\title{
User-centric Networking: Cooperation in Wireless Networks
}

\author{
Rute Sofia ${ }^{\star 1}$, Paulo Mendes ${ }^{1}$, Huiling Zhu², Alessandro Bogliolo ${ }^{3}$, Fikret \\ Sivrikaya ${ }^{4}$, Paolo di Francesco ${ }^{5}$ \\ 1 COPELABS, University Lusófona, Portugal \\ rute. sofia, paulo.mendes@ulus of ona.pt \\ ${ }^{2}$ University of Kent, United Kingdom \\ h.zhu@ac. kent.uk \\ 3 University of Urbino Italy \\ alessandro.bogliolo@uniurb.it \\ 4 DAI-Labor, Technical University of Berlin, Germany \\ fikret.sivrikaya@dai-labor.de \\ 5 Level7 S.p.A., Italy \\ paolo.difrancesco@level7.it

\begin{abstract}
This chapter addresses cooperation in wireless networks, based on the recent, self-organizing paradigm of User-centric Networking. In user-centric networking, the Internet user controls and carries networking wireless objects, usually located in customer premises. Some of such objects integrate functionality that is today part of the network core, such as mobility management, or resource management. As such functionality is placed closer to the Internet user, this stakeholder becomes more than a simple consumer of networking services, being a key element in Internet connectivity models, as well as in networking topology. Intrinsic to the notion of user-centric networking is the idea of resource sharing via cooperative elements, based on specific sharing incentives. The network dynamics are affected by such user empowerment in several ways. Devices on the network have limited capabilities such as energy or storage and change location frequently following their human carriers social behavior. These aspects drastically affect the Internet architectural design and introduces the need to revisit networking paradigms so that part of the networking functionality becomes closer and controllable by the Internet end-user, in a way that is beneficial to all Internet stakeholders.

The chapter provides notions and models concerning user-centric networking, as well as notions and models directed to user-centricity in the context of wireless network. We also include recent operational data derived from available user-centric networking pilots, as well as a market analysis with a wholesale model analysis example.
\end{abstract}

\footnotetext{
* Corresponding author. COPELABS, University Lusófona, Building U First Floor, Campo Grande 388, 1749-024 Lisbon, Portugal. 


\section{Introduction}

The flexibility inherent to wireless technologies is giving rise to new types of access networks and allowing the Internet to expand in a user-centric way. This is particularly relevant if one considers that wireless technologies such as Wireless Fidelity (Wi-Fi) are preferential solutions to complement Internet access broadband technologies, forming the last hop to the end-user. The density derived from such deployment, in particular in urban environments, must be considered when developing future Internet networking architectures. By working diversity and self-organization aspects, such wireless local-loop could then reach rates closer to the ones provided by current access technologies. In this line of research, the backbone infrastructure gets expanded via low-cost wireless technologies that embody a multi-operator model, i.e., a local-loop that is established with devices owned or carried by Internet end-users, or regular citizens, being the common interest of such elements the usage of shared resources, backed up by specific cooperation incentives and "good behavior" rules.

User-centric networks (UCNs) [1,9] are wireless networking architectures that integrate a self-organizing, even viral behavior in terms of connectivity models and topology, as these two aspects are developed based on the willingness of the Internet end-user to trade resources and services. In UCNs, users own devices that are part of the network. Therefore, UCNs exhibit a highly dynamic topological behavior, as the objects that compose the network roam frequently, and are in some cases solely controlled by the Internet end-user (e.g. a residential Access Point, AP; a smartphone).

In the networking models that UCNs integrate, the end-user is one of the Internet stakeholders, ceasing to simply be a consumer of Internet services (be it connectivity or content). The user becomes an active hop of the connectivity distribution chain. Such empowerment is a natural step of the Internet architectural evolution, as in Internet services, a similar paradigm shift has already emerged as a wave of open-source software and of new licensing models which culminated in the Creative Commons (CC) licensing. CC licensing allows authors to define the details of licensing rights regarding attribution, commercialization, derivative works, as well as distribution. In the beginning, CC licenses were used only in blogs or Web sites such as Flickr; today the Internet holds millions of sites whose content is protected under CC. This means that Internet users are no longer mere consumers.

The grassroots movement that was the basis for Web2.0 and also the key aspect in CC licensing is today being applied to the networking layers of the OSI protocol stack, e.g. via Software Defined Networking (SDN), and thus create opportunities to further evolve the Internet value chain. This is already happening both from a commercial and non-commercial perspective. For instance, current commercial examples of UCNs relate to the initiative of companies such as FON, OpenSpark. From a non-commercial perspective we assist to a number of usercentric or community initiatives (e.g. Freifunk, CUWin) which have as main purpose to assist in the development of Do-it-Yourself Networks (DIYN) based on Wi-Fi technology, always as a way to expand already existing/subscribed 
Internet connectivity. It should be noticed that such non-commercial initiatives are completely user-centric in the sense that it is up to the user not only to assist in building the last hop infrastructure, but also up to the end-user to control such last-hop architecture.

All of the paradigm shifts that we are witnessing are based upon a specific form of cooperation between end-users towards network access or towards Internet services/providers. Cooperation, as well as cooperation incentives, is therefore modeling a new category of Internet community and impacting social and business behavior. However, technical limitations of today's technologies, as well as a lack of understanding on how such micro business models may evolve and impact current Internet wholesale models still undermine the potential impact of networks where the user becomes an active link in the provision chain. There is neither a clear modeling of incentives nor clear mechanisms to develop cooperation incentives on the fly, incentives which are prerequisite to the growth of such types of networks.

Such variability is highly dependent on the Internet end-user social behavior and is currently dictating networking dynamics. For instance, users that have similar daily routines carry devices that can connect among themselves, with some level of stability. By analyzing and integrating networking metrics derived from social behavior, e.g. trust, it is feasible to assist the user in moving beyond the consumer role, to become a prosumer at the networking level.

This chapter is focused on UCNs and explores features and concepts of cooperation in heterogeneous wireless networks. The chapter gives insight not only to the most technological advances of UCNs, as well as onto the market aspects concerning UCN usage. The chapter covers also different aspects concerning cooperation incentives, which are essential to assist UCNs in becoming adequately integrated into the existing infrastructures.

The work and research that the chapter is focused upon derives from the research developed in the context of the European Project ULOOP (User-centric Wireless Local Loop) [9]. ULOOP worked several aspects of UCNs, both from a technical and socio-economic perspective, having as main goal to assist a robust and yet self-organizing deployment of UCNs.

The chapter is organized as follows. Section 2 goes over UCNs notions and terminology, covering the UCN model main functional blocks; live examples, as well as a socio-economic feasibility analysis for the ULOOP UCN model. Section 3 goes over cooperation aspects in UCNs, namely, incentives and crediting aspects, as well as explaining models. Section 4 is dedicated to operational aspects, and describes results of UCN live usage derived from the ULOOP pilot, which is a tool that is open to the global community. Section $\mathrm{xx}$ is dedicated to a market analysis of UCNs, providing an exercise for a wholesale model, based on two live UCN operators experience, FON, and ZON. The chapter concludes in section 6 , providing guidelines for future work. 


\section{UCN Background}

UCNs relate to a recent trend in spontaneous wireless deployments where individual users or communities of users share subscribed access in exchange of specific incentives. In literature, names that relate with the UCN concept are personal hotspot, spontaneous user-centric networks.

UCNs envision increasing the potential of the Internet by devising communication and networking technologies to support the creation of techno-social communities, providing a combination of information, communication and human elements, by incorporating social aspects in the way connectivity and routing models are addressed. As UCNs rely on communication opportunities, such as sharing of Internet access and relaying of available resources, cost reduction is expected, while extending the capillarity of current access networks. Shared services provide the means to consider new, community-oriented business models for both the Internet end-user, and access operators.

UCNs explore and integrate functional solutions to allow user-centric wireless (Wi-Fi) local loops to form and to develop in an autonomic and user-friendly manner. User-centric refers to a community model which extends the reach of a high rate, multi-access broadband backbone by means of communication opportunities provided by end-users, based upon cooperation incentives. Such incentives may relate to an individual or a community of individuals, as well as to access stakeholders. Moreover, user-centricity can be discussed from two different perspectives. Firstly, the user is in power of assisting the network in terms not only of its deployment, but also of its proliferation. Secondly, services to be provided by the end-user are assisted by an access infrastructure that is engineered towards assisting the user in terms of Quality of Experience (QoE). In regards to the first aspect, deployment refers to assisting in sharing equipment that makes the network scale. Deployment per se does not suffice for this type of architectures to grow.

\subsection{Notions and Terminology}

In UCNs, there are two fundamental networking roles: node and gateway. A UCN node concerns a role (software functionality) that a wireless capable device takes. Concrete examples of nodes can be specific user equipment, access points, or even some management server. A UCN gateway is a role (software functionality) that reflects an operational behavior making a UCN node capable of acting as a mediator between UCN systems and non-UCN systems - the outside world. The gateway role may or may not be owned and controlled by a UCN user; it may also or only be controlled by an access operator. The key differentiating factor of the role of gateway, in contrast to a regular UCN node, is the operational intelligence and mediation capability. Similarly to UCN nodes, the UCN gateway functionality may reside in the user-equipment, in APs, or even in the access network. Hence, they exhibit a feature that is key in user-centric environments: their behavior as part of the network is expected to be highly variable. Gateways 
will be active or inactive based on several conditions such as users' wishes and network load.

As previously mentioned, each UCN node has a unique owner, micro-provider $(M P)$, assigned. An owner is an entity (end-user, operator, virtual operator) that is responsible for any actions concerning his/her device. The term "responsible" reflects liability, i.e., from an operator's perspective the owner is the single responsible for the adequate/inadequate usage of the user's device within a specific, trust-bounded community.

A community in UCN is a set of UCN nodes that hold common interests (such as sharing connectivity or resources / peripherals) at some instant in time and space. In other words, nodes exhibit a space and time correlation that is the basis to establish a robust connectivity model. This is expected to be extrapolated by adequately modeling trust associations between nodes. We highlight that the notion of community does not have any relation whatsoever to an Online Social Network (OSN), nor even to some specific OSN subset.

An interest is here defined as a parameter capable of providing a measure (cost) of the "attention" of a node towards a specific location in a specific time instant. In other words, an interest is a parameter that provides a node with a measure of a specific time and space correlation. For instance, assuming that a user goes each Saturday morning to the coffee-shop on the neighborhood corner, an interest here could be "having a coffee". Other users in the same location (exhibiting a similar time and space correlation) are in the same place during an overlapping period of time. They all share an interest as they are all collocated in the same location for a specific period of time. The shared interest here is: attending the same coffee-shop. Therefore, owners may be complete strangers and yet, connectivity may be set across the devices, based on parameters such as specific Quality of Experience (QoE) metrics; node movement history; roaming and service sharing patterns.

In terms of generic model, UCN stakeholders are Internet users and Internet providers. These two stakeholders can, however, assume several of four main roles: user; micro-provider; provider; Over-the-Top (OTT) service provider.

The user is a role assigned to an entity that wants to use shared services at some instant in time and space. The micro-provider is a role assigned to an entity that is willing to share, at some instant in time and space, resources in exchange of specific cooperation incentives, or rewards. The provider is a role assigned to an entity that is responsible for direct Internet access, while the usual Over the Top (OTT) role relates with management of credentials or trust circles; initial authentication; gateway registration. As a coordinating role, the OTT does not have any impact on the way traffic is transmitted in the communities or across the Internet. Moreover, the OTT does not account for any end-to-end measures, such as data privacy, liability of the traffic source(s), or traceability.

The roles of UCN stakeholders (users, and providers) and their relationships shape the Internet design, as these relationships impact the communication in ways that were not foreseen, e.g., by placing both the upstream (from user to network) and downstream (from network to user) flows at an equal level. 
is that despite the fact that spectrum abounds, Signal to Noise Ratio (SNR) can be very low in some areas (known as grey areas).

As for Community 2, it stands for a mesh network also interconnected to the same LTE provider. There is no strict relation between a community and a geographic location.

Maria is a user in Community 1 carrying her Android smart-phone (User Equipment, UE). Maria's UE selects a specific gateway (AP or UE) to be associated with in a certain location. After the reception of her association request, the gateway broadcasts a query both via the Wi-Fi interface and the LTE interface (to reach the backend) in order to figure out whether Maria is or not an authorized and trusted user. At the same time, the chosen gateway also triggers an adequate gateway selection mechanism that takes into consideration not only Maria's expectations, but also the potential overlap and electromagnetic noise in the area, as well as the optimization of the load across the entire network. While roaming in Community 1, the gateway onto which Maria's UE is currently associated detects that she is on the move (e.g. due to SNR variations) and immediately attempts to estimate/anticipate a potential new anchor for connectivity (new gateway). Upon agreement between the gateways, Maria's $\mathrm{UE}$ is automatically attached to a new gateway which can fulfill Maria's service expectations.

Tom, another user of Community 1, is in a grey area. His device realizes that Maria's device allows connectivity relaying and therefore Tom's device triggers a request to connect to Maria's device. Maria allows other users with whom her device does not yet have a trust association established to interconnect by providing them a small amount of resources based on specific QoE requirements (e.g. only if her UE has enough battery level and up to $20 \%$ of Maria's link capacity). Therefore, Maria and Tom's UE automatically negotiate connectivity and Tom goes online through Maria's device. Michael, another Community 1 user, is a subscriber of a network operator different than the one Maria is subscribed to and also belongs to Community 1 . Given that they share interests in the context of Community 1, Michael and Maria are able to connect and exchange data directly, without going through their respective operators. Moreover, provided that there is such an available device, Michael can also profit from the Internet access while in Community 1. Every time Michael is within the coverage of Community 1 devices, his UE handovers from the $3 \mathrm{G}$ network to community 1 (through Maria's UE). As one of the services provided by the network, all the communication between Michael and other users inside Community 1 is performed locally, including voice and video calls, and thus, for Michael, this means that his traffic is offloaded from the $3 \mathrm{G}$ network to the cloud. Whenever Michael leaves community 1 area, his UE handovers back to the $3 \mathrm{G}$ network. Thanks to the resource optimization and load-balancing features of gateways within Community 1 continuously exchange data and thus offload / transfer some UE's to other elected gateways. Besides community 1, a second group of users belonging to Community 2 gets information about data being shared in Community 1 (e.g. through the backend system). The second group is located in Paris, at Bob's 
place. Bob is using a tethered Android powered smart phone to connect to the LTE network and then uses the UCN functionality on the phone, via Wi-Fi, to share Internet access.

A second applicability case is provided in Fig. 1 (b) , where from an end-toend perspective a single community is illustrated. Community 4 is connected to the Internet by means of a fixed operator (carrier-grade Ethernet/DSL).

The last hop to the user is Wi-Fi based. We also highlight that the number of UCN communities can profit from services that other users share. In this example the ultimate goal is not to expand coverage but instead to consider ULOOP functionality as an enabling technology platform for cooperative data dissemination. In regular deployments, such data cannot be available, as it is simply the result of a cooperative effort based on a self-organizing system. Moreover, end-user devices that are UCN enabled may be able to gather open data (data collected from the users' surrounding environment). This is, for instance, the case of Maria, who needs to print her boarding pass at an airport with Wi-Fi coverage. Due to the UCN functionality implemented in gateways and also provided directly by other users, Maria can print her boarding pass through John's device, a user that Maria's device trusts through a bi-directional trust association.

In Community 4, UCN functionality tracks user expectations and service response. Therefore, users providing expanded coverage have feedback about their resource usage on-the-fly. Moreover, the users are provided with incentives for sharing, e.g. more bandwidth in exchange of receiving some advertisement. Such tracking/monitoring can be performed based on the CPE (UE and gateway) or directly via UE. Moreover, such tracking relates to information that is not personal and that the user always acknowledges to provide beforehand. In this use-case, tracked data does impose neither any confidentiality nor privacy risk for the user. The UE serves the purpose of being part of the data dissemination towards users that share some form of interest, or for which there is some interconnection due to a dynamically established trust circle.

\subsection{Live Examples}

To better understand what may be the impact of UCNs over the Internet, we have analyzed some of the most representative UCN examples as of today, and categorized them into five different sets: hotspot UCNs; mesh UCNs; Social networking UCNs; Mobile/Provider UCNs [26]. This section summarizes our findings, to provide some understanding concerning the trend of UCNs, and the cooperation aspects behind such trend.

The existing UCNshave been analyzed and grouped into different categories according to their operation, and the proposed categories are illustrated in Fig. 2. As illustrated we consider five main models to categorize living examples of UCNs.

Hotspot UCNs represent the majority of UCNs around us. In this category, UCNs are built upon existing Wi-Fi hotspots. Hence, hotspot UCNs are based on Wi-Fi infrastructure-mode, where an $A P$ mediates all communication to and from a set of end-user devices. Users adhere to hotspot UCN models due to 

roaming incentives: a user shares his/her Internet access subscription in exchange
of roaming across other hotspots shared within the same community.
Mesh UCNs are possibly the oldest UCN category. It should be noticed that
UCNs based on mesh relate only to user-centric mesh networks, given that com-
munities of users autonomously deploy the network. The wireless infrastructure
is often deployed by the community to allow Internet expansion in relatively
large areas. Incentives relate to low-cost Internet expansion (capillarity) and not
so much with roaming. roaming incentives: a user shares his/her Internet access subscription in exchange
of roaming across other hotspots shared within the same community.
Mesh UCNs are possibly the oldest UCN category. It should be noticed that
UCNs based on mesh relate only to user-centric mesh networks, given that com-
munities of users autonomously deploy the network. The wireless infrastructure
is often deployed by the community to allow Internet expansion in relatively
large areas. Incentives relate to low-cost Internet expansion (capillarity) and not
so much with roaming. roaming incentives: a user shares his/her Internet access subscription in exchange
of roaming across other hotspots shared within the same community.
Mesh UCNs are possibly the oldest UCN category. It should be noticed that
UCNs based on mesh relate only to user-centric mesh networks, given that com-
munities of users autonomously deploy the network. The wireless infrastructure
is often deployed by the community to allow Internet expansion in relatively
large areas. Incentives relate to low-cost Internet expansion (capillarity) and not
so much with roaming. roaming incentives: a user shares his/her Internet access subscription in exchange
of roaming across other hotspots shared within the same community.
Mesh UCNs are possibly the oldest UCN category. It should be noticed that
UCNs based on mesh relate only to user-centric mesh networks, given that com-
munities of users autonomously deploy the network. The wireless infrastructure
is often deployed by the community to allow Internet expansion in relatively
large areas. Incentives relate to low-cost Internet expansion (capillarity) and not
so much with roaming. roaming incentives: a user shares his/her Internet access subscription in exchange
of roaming across other hotspots shared within the same community.
Mesh UCNs are possibly the oldest UCN category. It should be noticed that
UCNs based on mesh relate only to user-centric mesh networks, given that com-
munities of users autonomously deploy the network. The wireless infrastructure
is often deployed by the community to allow Internet expansion in relatively
large areas. Incentives relate to low-cost Internet expansion (capillarity) and not
so much with roaming. roaming incentives: a user shares his/her Internet access subscription in exchange
of roaming across other hotspots shared within the same community.
Mesh UCNs are possibly the oldest UCN category. It should be noticed that
UCNs based on mesh relate only to user-centric mesh networks, given that com-
munities of users autonomously deploy the network. The wireless infrastructure
is often deployed by the community to allow Internet expansion in relatively
large areas. Incentives relate to low-cost Internet expansion (capillarity) and not
so much with roaming. roaming incentives: a user shares his/her Internet access subscription in exchange
of roaming across other hotspots shared within the same community.
Mesh UCNs are possibly the oldest UCN category. It should be noticed that
UCNs based on mesh relate only to user-centric mesh networks, given that com-
munities of users autonomously deploy the network. The wireless infrastructure
is often deployed by the community to allow Internet expansion in relatively
large areas. Incentives relate to low-cost Internet expansion (capillarity) and not
so much with roaming. roaming incentives: a user shares his/her Internet access subscription in exchange
of roaming across other hotspots shared within the same community.
Mesh UCNs are possibly the oldest UCN category. It should be noticed that
UCNs based on mesh relate only to user-centric mesh networks, given that com-
munities of users autonomously deploy the network. The wireless infrastructure
is often deployed by the community to allow Internet expansion in relatively
large areas. Incentives relate to low-cost Internet expansion (capillarity) and not
so much with roaming.

Social networking UCNs rely on social networking to seamlessly distribute Wi-Fi credentials and to allow UCN expansion. A specific suer, micro-provider, shares Internet access based upon credentials that he/she controls and in situations where the gateway can be simultaneously used by different users, as happens in a household.
Mobile/provider UCNs are the most recent UCN category. The potential of
this type of UCNs is still to be unveiled but what is clear is that the solu-
tions here described will drive the deployment of UCNs. In this category, the
network is formed anytime/anywhere by users according to their needs and fol-
lowing the subscription rules of their cellular providers. Mobile-based UCNs are
often provided by operators due to offloading reasons, as a way to lower Capital
EXpenditures (CAPEX). Mobile/provider UCNs are the most recent UCN category. The potential of
his type of UCNs is still to be unveiled but what is clear is that the solu-
ions here described will drive the deployment of UCNs. In this category, the
network is formed anytime/anywhere by users according to their needs and fol-
owing the subscription rules of their cellular providers. Mobile-based UCNs are
often provided by operators due to offloading reasons, as a way to lower Capital
EXpenditures (CAPEX). happens in a household.
Mobile/provider UCNs are the most recent UCN category. The potential of
this type of UCNs is still to be unveiled but what is clear is that the solu-
tions here described will drive the deployment of UCNs. In this category, the
network is formed anytime/anywhere by users according to their needs and fol-
lowing the subscription rules of their cellular providers. Mobile-based UCNs are
often provided by operators due to offloading reasons, as a way to lower Capital
EXpenditures (CAPEX). happens in a household.
Mobile/provider UCNs are the most recent UCN category. The potential of
this type of UCNs is still to be unveiled but what is clear is that the solu-
tions here described will drive the deployment of UCNs. In this category, the
network is formed anytime/anywhere by users according to their needs and fol-
lowing the subscription rules of their cellular providers. Mobile-based UCNs are
often provided by operators due to offloading reasons, as a way to lower Capital
EXpenditures (CAPEX). happens in a household.
Mobile/provider UCNs are the most recent UCN category. The potential of
this type of UCNs is still to be unveiled but what is clear is that the solu-
tions here described will drive the deployment of UCNs. In this category, the
network is formed anytime/anywhere by users according to their needs and fol-
lowing the subscription rules of their cellular providers. Mobile-based UCNs are
often provided by operators due to offloading reasons, as a way to lower Capital
EXpenditures (CAPEX). happens in a household.
Mobile/provider UCNs are the most recent UCN category. The potential of
this type of UCNs is still to be unveiled but what is clear is that the solu-
tions here described will drive the deployment of UCNs. In this category, the
network is formed anytime/anywhere by users according to their needs and fol-
lowing the subscription rules of their cellular providers. Mobile-based UCNs are
often provided by operators due to offloading reasons, as a way to lower Capital
EXpenditures (CAPEX). happens in a household.
Mobile/provider UCNs are the most recent UCN category. The potential of
this type of UCNs is still to be unveiled but what is clear is that the solu-
tions here described will drive the deployment of UCNs. In this category, the
network is formed anytime/anywhere by users according to their needs and fol-
lowing the subscription rules of their cellular providers. Mobile-based UCNs are
often provided by operators due to offloading reasons, as a way to lower Capital
EXpenditures (CAPEX). happens in a household.
Mobile/provider UCNs are the most recent UCN category. The potential of
this type of UCNs is still to be unveiled but what is clear is that the solu-
tions here described will drive the deployment of UCNs. In this category, the
network is formed anytime/anywhere by users according to their needs and fol-
lowing the subscription rules of their cellular providers. Mobile-based UCNs are
often provided by operators due to offloading reasons, as a way to lower Capital
EXpenditures (CAPEX).

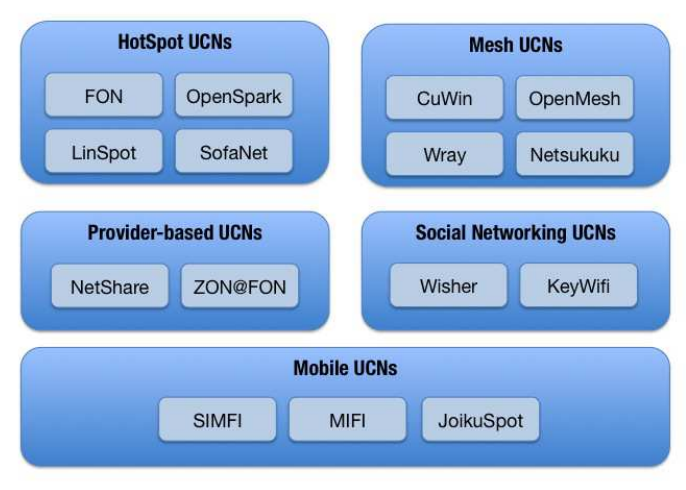

Fig. 2: UCN live examples categorization.

\footnotetext{
Let us start by explaining the main differences in terms of sharing capability
and where the management resides. For instance, in the UCN hotspot model,
the OTT role is usually a role of a provider. The same functionality is provided
by the OTT in the mesh-based model, but in this case the OTT corresponds to
a community of users. Similarly, in the social networking category, the OTT is
also a specific entity but its main responsibility is to ensure a secure exchange

Let us start by explaining the main differences in terms of sharing capability
and where the management resides. For instance, in the UCN hotspot model,
the OTT role is usually a role of a provider. The same functionality is provided
by the OTT in the mesh-based model, but in this case the OTT corresponds to
a community of users. Similarly, in the social networking category, the OTT is
also a specific entity but its main responsibility is to ensure a secure exchange

Let us start by explaining the main differences in terms of sharing capability
and where the management resides. For instance, in the UCN hotspot model,
the OTT role is usually a role of a provider. The same functionality is provided
by the OTT in the mesh-based model, but in this case the OTT corresponds to
a community of users. Similarly, in the social networking category, the OTT is
also a specific entity but its main responsibility is to ensure a secure exchange

Let us start by explaining the main differences in terms of sharing capability
and where the management resides. For instance, in the UCN hotspot model,
the OTT role is usually a role of a provider. The same functionality is provided
by the OTT in the mesh-based model, but in this case the OTT corresponds to
a community of users. Similarly, in the social networking category, the OTT is
also a specific entity but its main responsibility is to ensure a secure exchange

Let us start by explaining the main differences in terms of sharing capability
and where the management resides. For instance, in the UCN hotspot model,
the OTT role is usually a role of a provider. The same functionality is provided
by the OTT in the mesh-based model, but in this case the OTT corresponds to
a community of users. Similarly, in the social networking category, the OTT is
also a specific entity but its main responsibility is to ensure a secure exchange

Let us start by explaining the main differences in terms of sharing capability
and where the management resides. For instance, in the UCN hotspot model,
the OTT role is usually a role of a provider. The same functionality is provided
by the OTT in the mesh-based model, but in this case the OTT corresponds to
a community of users. Similarly, in the social networking category, the OTT is
also a specific entity but its main responsibility is to ensure a secure exchange Let us start by explaining the main differences in terms of sharing capability
and where the management resides. For instance, in the UCN hotspot model,
the OTT role is usually a role of a provider. The same functionality is provided
by the OTT in the mesh-based model, but in this case the OTT corresponds to
a community of users. Similarly, in the social networking category, the OTT is
also a specific entity but its main responsibility is to ensure a secure exchange
}

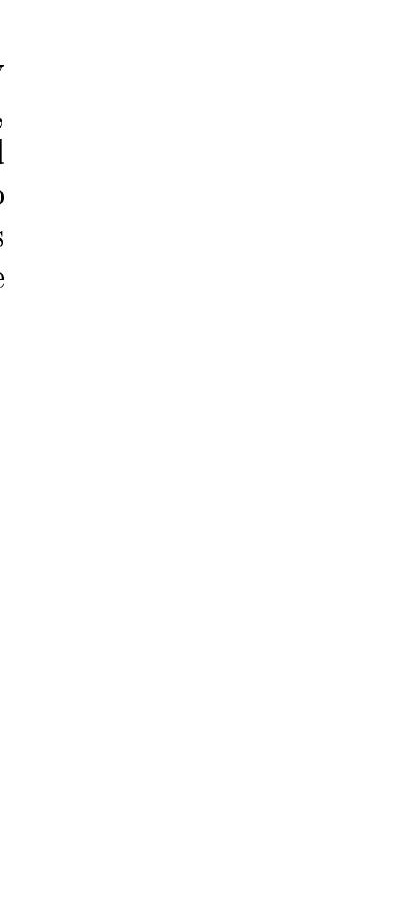

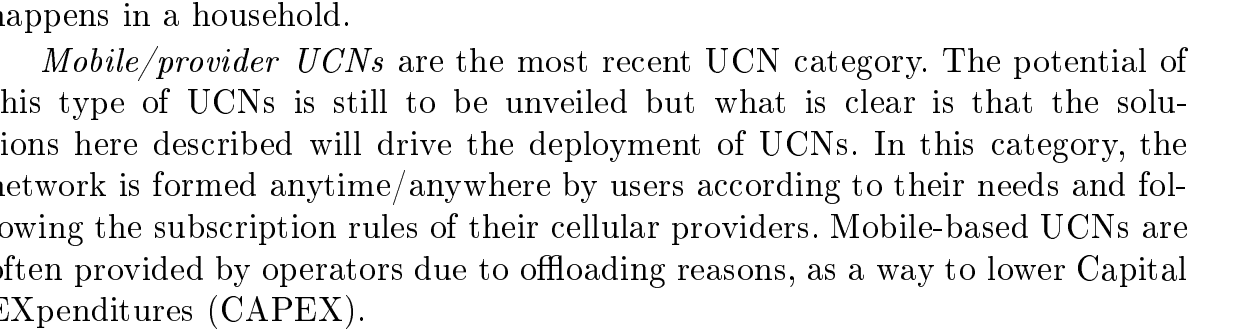

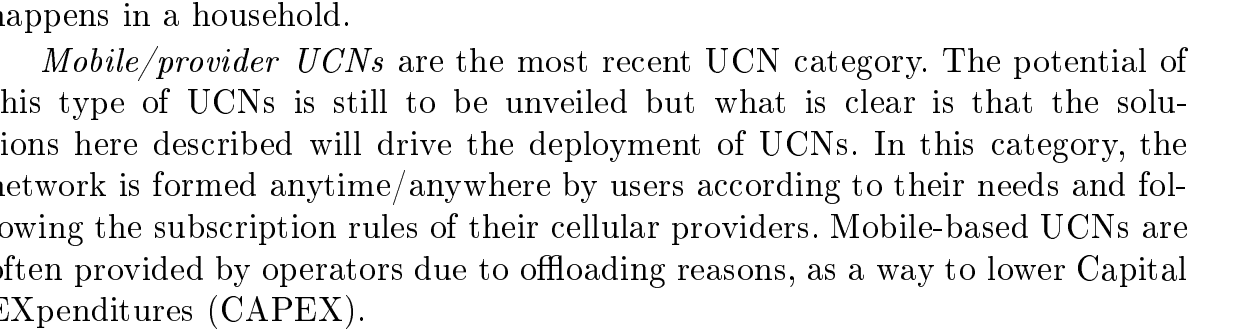


of credentials. In the case of the mobile-based and provider-based categories the OTT is actually the access operator.

The set of micro-providers in the hotspot, social networking, and mobile/provider models fairly corresponds to the global user database, given that all users must share access to obtain a specific benefit. While in the mesh and mobile categories the micro-provider set corresponds to a subset of the global community.

Changes in the micro-provider set are expected to be rare in most categories with the exception of the mobile-based category.

A relevant aspect to mention is the impact that changes on the micro-provider set may have on the overall network operation. In the hotspot model, and despite the fact that any user is a micro-provider, the impact of changes to the micro-provider set are not expected to affect significantly the network operation, given that such operation is tied to the Wi-Fi infrastructure mode, which splits the network operation into islands (hotspots). The same occurs in the social networking and provider-based categories. While in the mesh category, and despite the fact that micro-providers are a subset of the user universe, changes in such set are expected to bring high penalties to the network operation. This may possibly be counter-balanced due to the fact that most micro-providers are expected to exhibit a static behavior (roaming frequency may be low or scoped in nature).

In the mobile-based category changes to the micro-provider set will introduce high variability into the network, given that users are expected to roam frequently - such variability is tied to the mobility pattern of all users.

From a global perspective, today, and due to the fact that the micro-provider role is simply tied to the connectivity model, changes to the micro-provider set are not significant from an end-to-end Internet perspective. However, the micro-provider role is expected to evolve into a multi-user operation setting, where some forms of networking services, in particular in the control plane (e.g. mobility management) are to be sustained by the micro-provider in cooperation with the access, as starts to happen when smart APs (such as Femtocells) are deployed [27].

Let us now provide some considerations in terms of adoption incentives in UCNs, derived from the live example analyzis that we have performed. The promise of wider and free roaming is today the most common incentive. A second incentive observed is extra revenue or rebates for access cost. This incentive is more prominent in categories tied to the access stakeholders, e.g. provider-based categories, but it also appears in the social networking category. A third type of incentive is low-cost expansion of Internet access, and this is in fact the single incentive observed for examples that fall in the mesh category.

As these networks grow, incentives are also expected to evolve based upon new responsibilities that micro-providers may attain. For instance, incentives based on bandwidth tokens are feasible in a short-range time period, given that today such incentives are already present in a variety of collaborative tools, from an application layer perspective. 
An interesting technical aspect is that most of the categories do not consider resource management even in simple forms. The categories that integrate some form of resource management are the ones where the OTT is the provider mobile-based and provider-based categories -, which is somewhat obvious given that the control of the network is still centralized and hence, easier to perform: management of network resources in the mobile-based and the provider-based models is based on subscription rules. Resource management is, however, a field which is essential to develop, to assist UCN growth. Providing UCN stakeholders, in particular micro-providers, with the automatic capability to share bandwidth in a clever way is also an incentive that will be deployed in future models. Another relevant aspect to consider is that intelligent and dynamic resource management is a must to assist in preventing access technical infringements (e.g. going over the average rate stipulated in the Internet access subscription).

\subsection{UCN Model Functional Blocks}

UCNs assume that an existing infrastructure is available and that Internet users are willing to expand such infrastructure in a way that is user-friendly and selforganizing. UCNs assumes also that within specific trust circles some form of cooperation incentives can be provided in order for both the access and the end-user to cooperate and assist in further developing Internet architectures. In order for that to happen, our UCN model considers four main functional blocks: trust management and cooperation incentives; resource management; mobility aspects; and backward compatibility [27,33].

In this section we briefly explain the main functional blocks for a UCN model that has been conceived, validated, as well as implemented in the context of the European project ULOOP, being currently available to the community as opensource LGPLv3.0 software [3,2].

Trust Management and Cooperation Incentives Trust management and cooperation incentives relate with understanding how to define and build circles of trust on-the-fly. Such circles of trust are capable of sustaining an environment where stakeholders share some form of Internet resources in order to support the dynamic behavior of UCN. Trust management is based on reputation mechanisms able to identify end-user misbehavior and to address social aspects, e.g., the different types of levels of trust users may have in different communities (e.g., family, affiliation). In situations where the created network of trust is not enough to allow resources to be shared, devices are able to use a cooperation incentive scheme based on the transfer of credits directly proportional to the amount of shared resources [5].

Motivating Usage via Cooperation Cooperation incentives in UCN are considered both from a specific technology perspective, as well as from a business perspective. Technical incentives may relate to natural features of the technology that result in a win-win match when cooperation is applied. A concrete example 
of a technical incentive relates to potential improvements of the 802.11 MAC layer. UCN engineers the MAC layer in a way that mitigates problems related with low data rate stations. Hence, when low data rate stations and high data rate stations cooperate, all elements are expected to take some advantage of such cooperation. While as for a business incentive, we can think of a specific peering scheme that may assist the access operators in understanding how to obtain revenue based on UCN architectures.

As part of communities and also as individual nodes, cooperation must consider the willingness of owners/nodes in participating in communication. Willingness can be driven by different facts such as energy saving, low processing power, and/or lack of storage room. Although a node is not willing to share resources due to one of the aforementioned facts, the cooperation functionality should encourage such user in doing it so, as he/she can get an immediate return (e.g., more processing) while sharing that resource it has the most (e.g., storage). Instead of simply paying users with the same "currency", e.g., you get more bandwidth if you give more bandwidth, the cooperation functionality should reward involved entities with the type of resource the user wants and at the moment the user needs (i.e., immediately or later on).

Augmented Resource Management As UCN relies on wireless infrastructures that are often deployed in an ad-hoc way, resource management optimization is a key aspect to pursuit. UCN has as purpose to assist in developing robust and high debit wireless local-loops in a way that meets current broadband access technologies debit as possible, and in a way that reduces the chances for bottlenecks to occur. Throughput maximization is to be addressed across more than one hop by means of cooperative networking techniques of which one possibility is relaying. In regards to resource management, and to achieve a fair and selforganizing network operation, there are aspects to be looked for such as the need to adequately and dynamically be able to control growth of UCN communities; dynamic fluctuations of the network both in terms of traffic due to stations joining and leaving frequently, as well as due to the movement of stations. Another aspect that is considered crucial to look for is to develop cooperative and distributed mechanisms that assist the network in adequately selecting nodes that are willing to be micro-providers. Such selection is to be performed in a way that considers not only throughput maximization, but also the lowest-cost in terms of energy-efficiency.

Dealing with Frequent Roaming: Anchor Point Control and Movement Estimation UCN is based on the notion of users carrying (or owning) low-cost and limited capacity portable devices which are cooperative in nature and which extend the network in a user-centric way, not necessarily implying the support for networking services such as multi-hop routing. For instance, in UCNs transmission may simply be relayed based on simple mechanisms already existing in end-user devices. These emerging architectures therefore represent networks where the nodes that integrate the network are in fact end-user devices 
which may have additional storage capability and which may or may not sustain networking services. Such nodes, being carried by end-users exhibit a highly dynamic behavior. Nodes move frequently following social patterns and based on their carriers interests; inter-contact exchange is the basis for the definition of connectivity models as well as data transmission. The network is also expected to frequently change (and even to experience frequent partitions) due to the fact that such nodes, being portable, are limited in terms of energy resources.

In terms of mobility and adding to the currently available solutions, UCN is focused on two main aspects: mobility tracking and estimation, as well as handover support. Ways of addressing patterns of node movement to estimate mobility patterns based on existing or novel social models is one aspect to be addressed [19]. The purpose is to assist in improving the underlying connectivity model, and hence overall network operation. Social mobility modeling is an aspect that assists in deriving algorithms and functionality that can anticipate the way nodes move based on analysis and tracking of node movement through time.

A final aspect to consider is to ensure that the functionality to be developed can assist in dealing with the unmanaged aspects of UCN architecture and should get rid of anchors in the network. This may be required, for instance, if a UCN community is not capable of providing a node with adequate mobility management e.g. due to trust aspects.

\subsection{Regulatory and Socio-economic Implications}

The potential of UCNs can be exploited both to complement broadband access (by increasing capillarity/coverage and by providing $3 \mathrm{G}$ to Wi-Fi offloading) and to implement a self-adapting territorial platform for collaborative data gathering and dissemination. These two broad application scenarios correspond to the usecases described in section 2.2 and which were considered in the context of the ULOOP project to establish realistic boundaries and requirements for UCNs.

In order to support the socio-economic analysis of both scenarios, the project considered several more specific "scenes" derived from each use-case [10].

Methodology to Analyze the Impact on Regulation UCNs are a relatively new concept which is not yet included in regulatory frameworks. Still, the impact on regulation is a determinant for the diffusion of any new technology or model. This is particularly true for community-scale models which need to reach a high penetration in order to trigger the positive externalities which can make them profitable. In order to evaluated the impact of UCNs both at a national and international level, the ULOOP project considered a methodology based on questionnaires and scoring sheets, questionnaire which is illustrated in Fig. 3. The questionnaire encompasses 14 questions covering the main relevant regulatory aspects, divided into five groups. Questions Q01 and Q02 are about the general principles of Net Neutrality and Universal Service, which might contribute to the diffusion of the UCN model, if properly adopted. Questions Q03 
to Q06 relate with electronic communications, with particular emphasis on the definitions and possible limitations which apply to "non/public networks", as opposed to public communication networks managed by operators and established service providers.

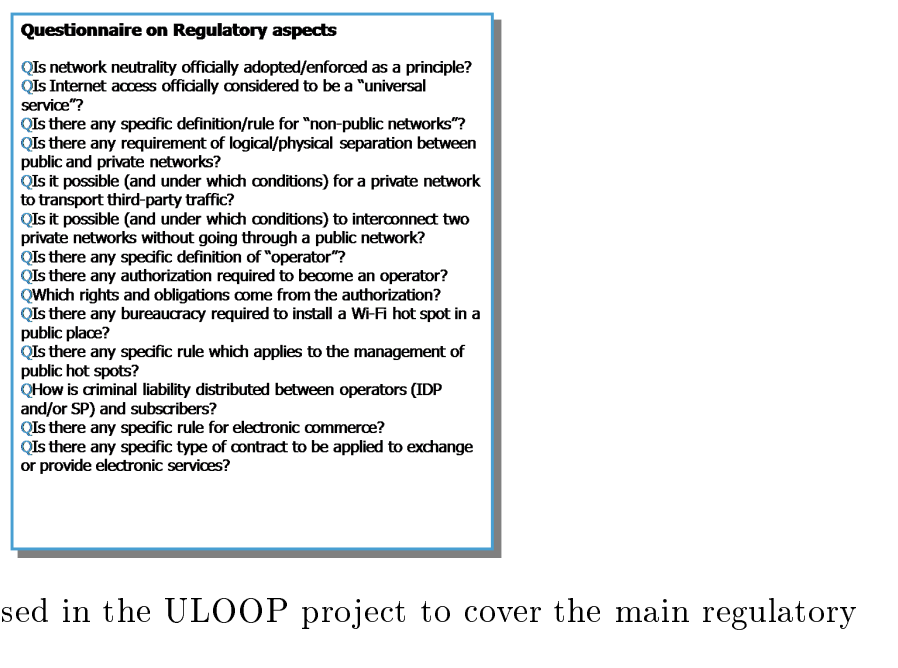

Fig. 3: Questionnaire used in the ULOOP project to cover the main regulatory aspects for UCNs.

Questions Q07 to Q09 cover the definition of "operator" and the exclusive rights and obligations coming from the so called general authorization, which

Socio-economic Analysis The socio-economic sustainability of a UCN de-
pends on the diffusion of UCN devices which, in its turn, depends on the capabil-
ity of the model to attract people. Hence, the sustainability analysis has to start
from the key features of a UCN, which have to be perceived as added-value or
differentiators to the stakeholders involved in the model. For the socio-economic
analysis we grouped the distinguishing features of UCNs into four main cate-
gories: resources; information; availability; protection. The scenes introduced to
represent the potential of the two use cases ULOOP-1 and ULOOP-2 were then
weighted in terms of each of the features described [10].

Socio-economic Analysis The socio-economic sustainability of a UCN de-
pends on the diffusion of UCN devices which, in its turn, depends on the capabil-
ity of the model to attract people. Hence, the sustainability analysis has to start
from the key features of a UCN, which have to be perceived as added-value or
differentiators to the stakeholders involved in the model. For the socio-economic
analysis we grouped the distinguishing features of UCNs into four main cate-
gories: resources; information; availability; protection. The scenes introduced to
represent the potential of the two use cases ULOOP-1 and ULOOP-2 were then
weighted in terms of each of the features described [10].

Socio-economic Analysis The socio-economic sustainability of a UCN de-
pends on the diffusion of UCN devices which, in its turn, depends on the capabil-
ity of the model to attract people. Hence, the sustainability analysis has to start
from the key features of a UCN, which have to be perceived as added-value or
differentiators to the stakeholders involved in the model. For the socio-economic
analysis we grouped the distinguishing features of UCNs into four main cate-
gories: resources; information; availability; protection. The scenes introduced to
represent the potential of the two use cases ULOOP-1 and ULOOP-2 were then
weighted in terms of each of the features described [10].

Socio-economic Analysis The socio-economic sustainability of a UCN de-
pends on the diffusion of UCN devices which, in its turn, depends on the capabil-
ity of the model to attract people. Hence, the sustainability analysis has to start
from the key features of a UCN, which have to be perceived as added-value or
differentiators to the stakeholders involved in the model. For the socio-economic
analysis we grouped the distinguishing features of UCNs into four main cate-
gories: resources; information; availability; protection. The scenes introduced to
represent the potential of the two use cases ULOOP-1 and ULOOP-2 were then
weighted in terms of each of the features described [10].

Socio-economic Analysis The socio-economic sustainability of a UCN de-
pends on the diffusion of UCN devices which, in its turn, depends on the capabil-
ity of the model to attract people. Hence, the sustainability analysis has to start
from the key features of a UCN, which have to be perceived as added-value or
differentiators to the stakeholders involved in the model. For the socio-economic
analysis we grouped the distinguishing features of UCNs into four main cate-
gories: resources; information; availability; protection. The scenes introduced to
represent the potential of the two use cases ULOOP-1 and ULOOP-2 were then
weighted in terms of each of the features described [10].

Socio-economic Analysis The socio-economic sustainability of a UCN de-
pends on the diffusion of UCN devices which, in its turn, depends on the capabil-
ity of the model to attract people. Hence, the sustainability analysis has to start
from the key features of a UCN, which have to be perceived as added-value or
differentiators to the stakeholders involved in the model. For the socio-economic
analysis we grouped the distinguishing features of UCNs into four main cate-
gories: resources; information; availability; protection. The scenes introduced to
represent the potential of the two use cases ULOOP-1 and ULOOP-2 were then
weighted in terms of each of the features described [10].

Socio-economic Analysis The socio-economic sustainability of a UCN de-
pends on the diffusion of UCN devices which, in its turn, depends on the capabil-
ity of the model to attract people. Hence, the sustainability analysis has to start
from the key features of a UCN, which have to be perceived as added-value or
differentiators to the stakeholders involved in the model. For the socio-economic
analysis we grouped the distinguishing features of UCNs into four main cate-
gories: resources; information; availability; protection. The scenes introduced to
represent the potential of the two use cases ULOOP-1 and ULOOP-2 were then
weighted in terms of each of the features described [10].

Socio-economic Analysis The socio-economic sustainability of a UCN de-
pends on the diffusion of UCN devices which, in its turn, depends on the capabil-
ity of the model to attract people. Hence, the sustainability analysis has to start
from the key features of a UCN, which have to be perceived as added-value or
differentiators to the stakeholders involved in the model. For the socio-economic
analysis we grouped the distinguishing features of UCNs into four main cate-
gories: resources; information; availability; protection. The scenes introduced to
represent the potential of the two use cases ULOOP-1 and ULOOP-2 were then
weighted in terms of each of the features described [10].

Socio-economic Analysis The socio-economic sustainability of a UCN de-
pends on the diffusion of UCN devices which, in its turn, depends on the capabil-
ity of the model to attract people. Hence, the sustainability analysis has to start
from the key features of a UCN, which have to be perceived as added-value or
differentiators to the stakeholders involved in the model. For the socio-economic
analysis we grouped the distinguishing features of UCNs into four main cate-
gories: resources; information; availability; protection. The scenes introduced to
represent the potential of the two use cases ULOOP-1 and ULOOP-2 were then
weighted in terms of each of the features described [10].

(1)

\footnotetext{
西
}

\begin{abstract}
are essential to understand whether and to what extent allow UCN users are
allowed to provide associated services and facilities without being established are essential to understand whether and to what extent allow UCN users are
allowed to provide associated services and facilities without being established service providers. Questions Q10 to Q12 concern the bureaucracy required to install/activate/manage a public Wi-Fi access point, which might limit the diffusion of UCNs. Questions Q13 and Q14 are about e-commerce regulation.

As for the scoring sheet, it considered the relevance of each question within the context of the two use-cases described in section 2.2. Answers to the questionnaire were collected via involvement of several European regulation authorities from several European Countries: Italy, Spain, France, Switzerland, and Finland [10].
\end{abstract} 然

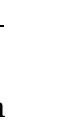
d

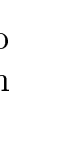


communication system can be improved. By leveraging the broadcast nature of the wireless channels, cooperative networking significantly improves system performance. While scientific results abound in what concerns cooperative networking, the feasibility of applying it beyond cooperative wireless transmissions is not yet fully understood.

One key aspect to address relates with incentive mechanisms to boost cooperation. Another key aspect relates with the potential compatibility of the developed cooperative networking mechanisms, across existing infrastructures. In the context of UCNs, a cooperative networking model must be based on effective incentives for cooperation, and on an efficient improvement of networking experience for all of the UCN model stakeholders.

\subsection{Cooperation and Sharing}

Cooperation is a central feature of user-centric systems aiming to compensate for the lack of a central and dedicated controlling entity. In spontaneous networks, such as UCNs, users need to cooperate in order to help one another aiming to mitigate the constraints posed by the dynamic networking environment. Such cooperation can be achieved by sharing available resources (e.g., bandwidth, processing power, memory, battery) and/or effort.

The success of a cooperation process may be diminished by the fact that users have full control on their devices and may try to maximize the benefits they get from the network. In general, the cooperative behaviour of a device will indeed result in an increase in resource consumption to take more than its fair share of a resource (e.g., network, CPU, storage). Knowing that mobile devices have scarce resources, each of these devices should better not cooperate from its point of view. Despite the potential advantages of enrolling in a cooperation process, it is imperative to give users the option to participate or not in cooperation. That is, user willingness to cooperate must be considered.

Three reasons may discourage willingness to participate in cooperation: i) lack of trust on other users, which can be mitigated by a mechanism able to manage trust; ii) users are running out of resources, which can be lessened if offering users resources that may improve (e.g., increase battery lifetime, processing power, storage room) their own operation; and iii) users' egoistic behaviour, that is easily diminished once users know they will have resources available upon their needs. However, independently of what is increasing/decreasing their willingness level, users will easily engage in cooperation if they know that they will get resources whenever they need them.

In what concerns the type of shared resources, related literature is usually focused on cooperation related with sharing of the same category of resources, e.g. storage by storage; processing by processing; roaming now by roaming later. However, it is envisioned that UCNs, being software based, provide the grounds to exchange resources based on different categories as this is a main feature to ensure an efficient usage of networking resources in wireless heterogeneous networks. This can be achieved via a cooperation model/mechanism that provides a 
direct exchange of networking resources, for instance, connectivity time against bandwidth, or networking storage vs. Quality of Service.

\section{In a UCN, cooperation incentives are implemented as a combination of: trust- based, in which incentives for cooperation are created by adopting policies that
are based on the threat of retaliation for non-cooperating nodes; reward-based,
in which nodes get a virtual payment for cooperating. Such incentives are used
to motivate nodes to cooperate in order to augment their networking experience.
The ULOOP project focus on the investigation of two cooperation mechanisms
to improve network efficiency: i) cooperation-based management of wireless re-
sources; ii) few-hop relaying. based, in which incentives for cooperation are created by adopting policies that
are based on the threat of retaliation for non-cooperating nodes; reward-based,
in which nodes get a virtual payment for cooperating. Such incentives are used
to motivate nodes to cooperate in order to augment their networking experience.
The ULOOP project focus on the investigation of two cooperation mechanisms
to improve network efficiency: i) cooperation-based management of wireless re-
sources; ii) few-hop relaying. based, in which incentives for cooperation are created by adopting policies that
are based on the threat of retaliation for non-cooperating nodes; reward-based,
in which nodes get a virtual payment for cooperating. Such incentives are used
to motivate nodes to cooperate in order to augment their networking experience.
The ULOOP project focus on the investigation of two cooperation mechanisms
to improve network efficiency: i) cooperation-based management of wireless re-
sources; ii) few-hop relaying. based, in which incentives for cooperation are created by adopting policies that
are based on the threat of retaliation for non-cooperating nodes; reward-based,
in which nodes get a virtual payment for cooperating. Such incentives are used
to motivate nodes to cooperate in order to augment their networking experience.
The ULOOP project focus on the investigation of two cooperation mechanisms
to improve network efficiency: i) cooperation-based management of wireless re-
sources; ii) few-hop relaying. based, in which incentives for cooperation are created by adopting policies that
are based on the threat of retaliation for non-cooperating nodes; reward-based,
in which nodes get a virtual payment for cooperating. Such incentives are used
to motivate nodes to cooperate in order to augment their networking experience.
The ULOOP project focus on the investigation of two cooperation mechanisms
to improve network efficiency: i) cooperation-based management of wireless re-
sources; ii) few-hop relaying. based, in which incentives for cooperation are created by adopting policies that
are based on the threat of retaliation for non-cooperating nodes; reward-based,
in which nodes get a virtual payment for cooperating. Such incentives are used
to motivate nodes to cooperate in order to augment their networking experience.
The ULOOP project focus on the investigation of two cooperation mechanisms
to improve network efficiency: i) cooperation-based management of wireless re-
sources; ii) few-hop relaying. based, in which incentives for cooperation are created by adopting policies that
are based on the threat of retaliation for non-cooperating nodes; reward-based,
in which nodes get a virtual payment for cooperating. Such incentives are used
to motivate nodes to cooperate in order to augment their networking experience.
The ULOOP project focus on the investigation of two cooperation mechanisms
to improve network efficiency: i) cooperation-based management of wireless re-
sources; ii) few-hop relaying. A Direct Exchange Cooperation Framework Model, USWAP As ex-
plained, UCNs are expected to profit from integrating a framework that can
assist direct exchange of different types of (networking) resources. To provide an
example, Fig. 5 contains several networking resources, some which are traffic-
related resources, while others are in categories such as storage, or time (e.g.
lifetime). The third column explains the mapping of the resource to one or more A Direct Exchange Cooperation Framework Model, USWAP As ex-
plained, UCNs are expected to profit from integrating a framework that can
assist direct exchange of different types of (networking) resources. To provide an
example, Fig. 5 contains several networking resources, some which are traffic-
related resources, while others are in categories such as storage, or time (e.g.
lifetime). The third column explains the mapping of the resource to one or more A Direct Exchange Cooperation Framework Model, USWAP As ex-
plained, UCNs are expected to profit from integrating a framework that can
assist direct exchange of different types of (networking) resources. To provide an
example, Fig. 5 contains several networking resources, some which are traffic-
related resources, while others are in categories such as storage, or time (e.g.
lifetime). The third column explains the mapping of the resource to one or more A Direct Exchange Cooperation Framework Model, USWAP As ex-
plained, UCNs are expected to profit from integrating a framework that can
assist direct exchange of different types of (networking) resources. To provide an
example, Fig. 5 contains several networking resources, some which are traffic-
related resources, while others are in categories such as storage, or time (e.g.
lifetime). The third column explains the mapping of the resource to one or more A Direct Exchange Cooperation Framework Model, USWAP As ex-
plained, UCNs are expected to profit from integrating a framework that can
assist direct exchange of different types of (networking) resources. To provide an
example, Fig. 5 contains several networking resources, some which are traffic-
related resources, while others are in categories such as storage, or time (e.g.
lifetime). The third column explains the mapping of the resource to one or more A Direct Exchange Cooperation Framework Model, USWAP As ex-
plained, UCNs are expected to profit from integrating a framework that can
assist direct exchange of different types of (networking) resources. To provide an
example, Fig. 5 contains several networking resources, some which are traffic-
related resources, while others are in categories such as storage, or time (e.g.
lifetime). The third column explains the mapping of the resource to one or more

(1)

(1)
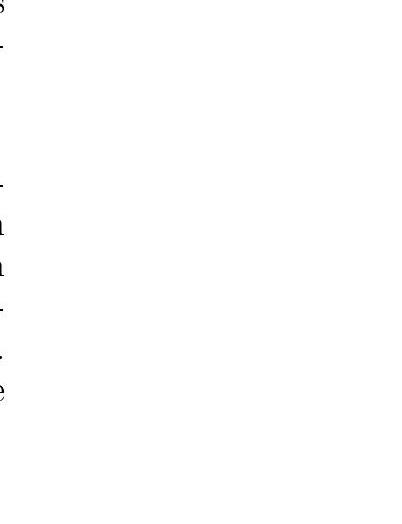

\subsection{Cooperation Models OSI Layers.}

\begin{tabular}{|c|c|c|}
\hline Resource & Description & Related OSI Layers \\
\hline Traffic rate & Number of bits sent per second. & OSI Layers 1, 2, 3 \\
\hline Connectivity & Internet acoess time (seconds). & OSI Layer 3 \\
\hline Coverage & $\begin{array}{l}\text { Radius of coverage, associated to } \\
\text { the signal power, and measured } \\
\text { e.g. by the SNRI. }\end{array}$ & OSI Layer 1,2 \\
\hline Storage & $\begin{array}{l}\text { Amount of disk to be shared e.g. } \\
\text { for the purpose of caching. } \\
\text { Measured in bytes. }\end{array}$ & OSI Layer 7 \\
\hline Lifetime & $\begin{array}{l}\text { Corresponds to the period of time } \\
\text { since a node (or network) } \\
\text { becomes active until the node } \\
\text { is said to be dead, i.e., from a } \\
\text { network perspective, the node } \\
\text { ceases to exist. Is assodiated to } \\
\text { the energy consumption of the } \\
\text { node (or network) }\end{array}$ & OSI Layer 1 \\
\hline Bandwidth & Wireless capacity chunk & OSI Layer2 1,2 \\
\hline $\begin{array}{l}\text { Frequency } \\
\text { chunk }\end{array}$ & $\begin{array}{l}\text { A sub-frequency range, or an } \\
\text { aggregation of sub-frequencies }\end{array}$ & OSI Layer 1 \\
\hline Duty cyde & $\begin{array}{l}\text { The time a node takes to serve a } \\
\text { set of requests }\end{array}$ & OSI Layer 2 \\
\hline Density & $\begin{array}{l}\text { Number of nodes in a network, or } \\
\text { neighbors of a specific node. The } \\
\text { higher that number, the more } \\
\text { dense the node is. }\end{array}$ & OSI Layers 1, 2 \\
\hline
\end{tabular}

Fig. 5: Potential networking resources to be considered for direct exchange.

In this section we describe a potential example of such a framework, the
USWAP - Cooperative Network Resources Swapping in User-centric Networks
software-defined framework, for which a high-level representation is provided in
Fig. 6, a software-defined solution that can run on UCN nodes. The modules rep-
resented shall reside on user space, OSI Layer 7 . The USWAP resource manager

In this section we describe a potential example of such a framework, the
USWAP - Cooperative Network Resources Swapping in User-centric Networks
software-defined framework, for which a high-level representation is provided in
Fig. 6, a software-defined solution that can run on UCN nodes. The modules rep-
resented shall reside on user space, OSI Layer 7. The USWAP resource manager

In this section we describe a potential example of such a framework, the
USWAP - Cooperative Network Resources Swapping in User-centric Networks
software-defined framework, for which a high-level representation is provided in
Fig. 6, a software-defined solution that can run on UCN nodes. The modules rep-
resented shall reside on user space, OSI Layer 7. The USWAP resource manager

In this section we describe a potential example of such a framework, the
USWAP - Cooperative Network Resources Swapping in User-centric Networks
software-defined framework, for which a high-level representation is provided in
Fig. 6, a software-defined solution that can run on UCN nodes. The modules rep-
resented shall reside on user space, OSI Layer 7. The USWAP resource manager In this section we describe a potential example of such a framework, the
USWAP - Cooperative Network Resources Swapping in User-centric Networks
software-defined framework, for which a high-level representation is provided in
Fig. 6, a software-defined solution that can run on UCN nodes. The modules rep-
resented shall reside on user space, OSI Layer 7 . The USWAP resource manager

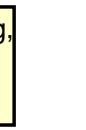

.

.

exchange.
framework, the
The modules rep-
Tis mource manager

. . 
(RM) is an entity based on the ULOOP Resource Management entity, extended via the inclusion of two additional modules: a Cooperative Resource Exchange (CRE) module, and the Self-organizing Swapping (SOS) entity. CRE deals with the optimization of the proposed exchanges, for instance, by considering relaying, or offloading to multiple nodes around (instead of onto a single node). The SOS entity is responsible for ensuring that the swapping done is beneficial both from a node and from a network perspective, to prevent nodes from becoming greedy or jeopardizing the whole system.

The USWAP Network Resource Exchange Mapper (NREM) is an abstraction entity that assists in the direct exchange of networking resources.

The USWAP Exchange Adaptation (EXA) entity is a module that assists the exchange, by considering algorithms that assist in a dynamic exchange. For instance, if a device intends to swap network storage per connectivity time, then this entity shall analyse the cost efficiency of accepting such a request. If there are different possibilities offered, this entity shall optimize and prioritize the exchange possibilites via the Policy Interface which shall be developed in a way that is easily extendable, e.g. XML.

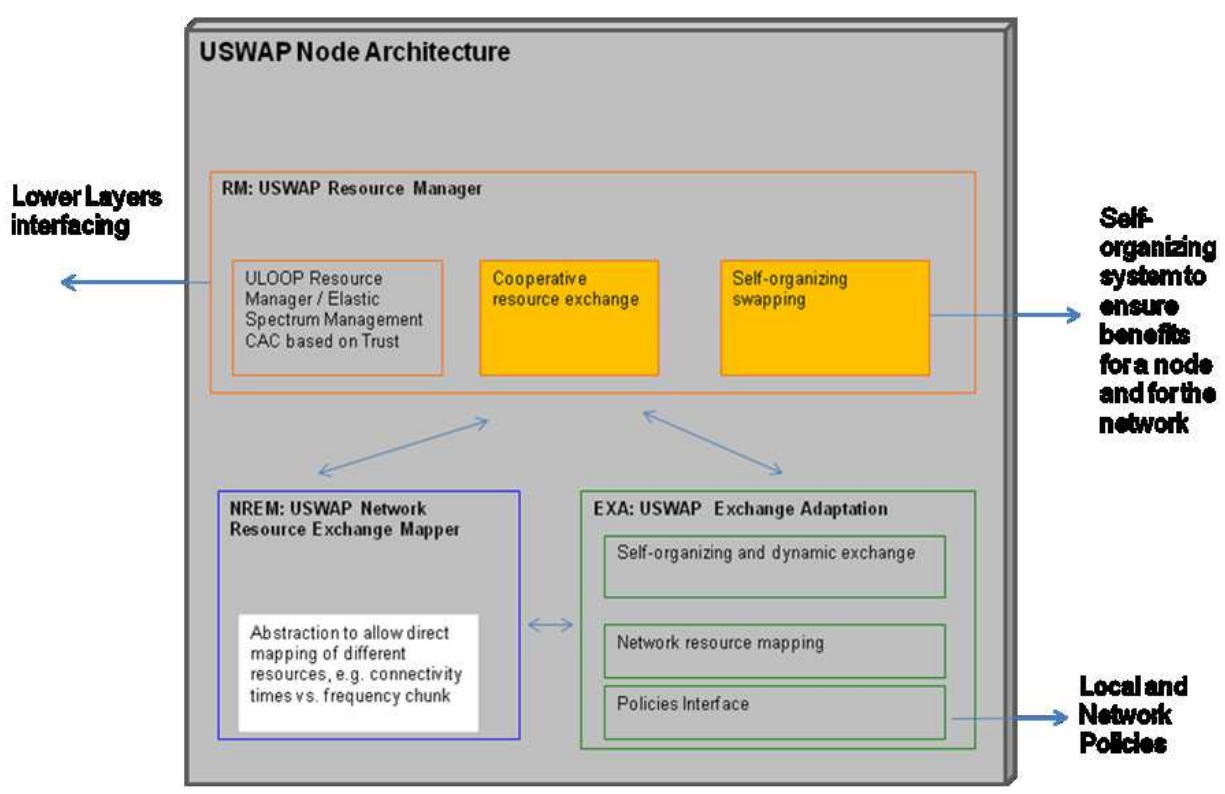

Fig. 6: Potential networking resources to be considered for direct exchange.

Let us provide an example considering two different USWAP nodes (e.g. an end-user device and an access point), which engage in active communication, to exchange resources. The UCN node wants to access the Internet freely for 60 minutes and in order to do so, is offering to swap two different types of networking 
resources: $200 \mathrm{Mb}$ of network storage, or $30 \mathrm{~m}$ of relaying to others. The AP, via e.g. a resource management entity, gets the request, and asks the NREM entity to wrap adequately the type of resources being offered. This wrapping implies that the NREM shall analyse whether or not the node contemplates the type of offer being done, via both local policies and eventually network policies. For instance, this may be a node that is not willing to consider network storage as a networking good. This mediation implies that both the NREM and EXA become involved in this process. Then, the EXA entity provides the adequate negotiation and adaptation, via self-organizing and cooperative mechanisms described in WP3, to ensure an optimal mapping of resources offered. EXA then provides an answer to RM, stating that the node accepts the offer of exchanging 200MB network storage per 60 minutes of connectivity. RM then replies to the requester, in order to trigger the regular connection process. This means that, for instance, RM . interfaces with the node captive portal to allow the requester to access the network in exchange of $30 \mathrm{~m}$ of connectivity.

Cooperation-based Resource Management The management of network resources takes advantage of the willingness that users have in cooperating, based on the mentioned two types of incentives: trust-based and reward-based. Cooperative based resource management has three components: call admission control, resource allocation and load balancing. Call admission control decides whether a new request from an end-user can be accepted or not. In one gateway, resource allocation assigns resources among all accepted and active end-users. Load-balancing can be used to react to congestion or to prevent congestion in the presence of a prediction mechanism. Call admission control in is innovative since once a request for specific resources arrives to a gateway, the gateway will take into consideration not only the available resources, but also the requesting end-user's credits, which are gained by the end-user through its previous cooperation and behavior (e.g. by sharing services and resources). The allocation of resources can, for instance, be done according to the type of requested resources (e.g. real-time vs non real-time) and the amount of credits own by the end-user (requester). Based on these two criteria, resources (e.g. bandwidth, power and bit rates) are allocated fairly among users aiming to guarantee the quality level required by them, while weighting users based on they cooperation level within the community. For instance, a user with a high quality demand with a cooperation deficit in a UCN community (few earned credits) may get fewer resources than another user with a lower quality demand, but with a higher cooperation index (more earned credits) in case of scarcity of resources. That is to say that resource allocation does not consider only the service quality obtained by each individual end-user, but also the whole performance of the community: network capacity can be maximized by increasing the incentives for nodes to share services and/or resources by providing them higher priority to access shared services/resources. In the presence of congestion, load balancing aims to provide a more efficient usage of resources by different gateways. A congested gateway may decide to shift traffic towards another gateway based on local measurements and by an- 
alyzing the measurements of neighbor gateways. The latter cooperate with the congested gateway by deciding to receive some of its traffic, since they will get extra credits for that. The decision to help balancing the load of a congested gateway is done based on a cooperation index, which is locally computed based on the analysis of the trade-off between accepting traffic from neighbors and the advantage of having more credits. Based on the list of neighbor gateways that are willing to cooperate with the congested gateway, the later will select a neighbor based on the trust level that the users, trying to access the congested gateway, have on the potential new gateway.

\section{Operational Aspects, the ULOOP Pilot}

The ULOOP project has deployed a model of UCNs and built a pilot composed of different sites each of which are described via Fig. 7[16]. Test sites are open to the global community and have been used during the project lifespan to test and to validate software developed. Pilot sites were used to gather results concerning operational UCN usage.

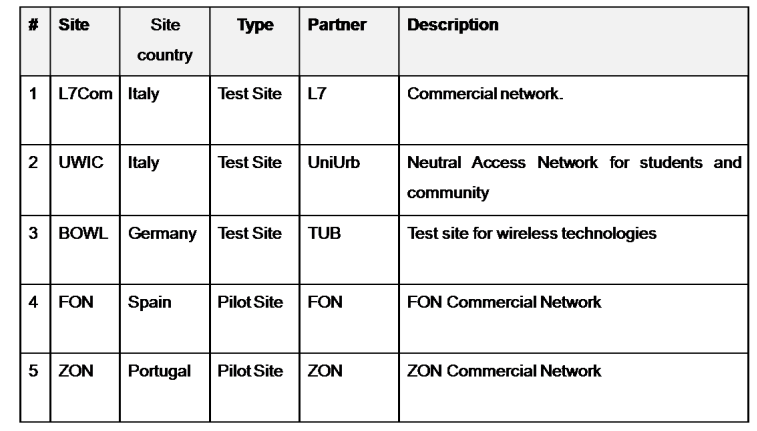

Fig. 7: ULOOP Pilot sites, where specific UCN functional blocks are available to the global community.

The ULOOP partners ZON and FON were responsible for setting up the pilot sites. These two entities have past experience working together, with FON's Community Wi-Fi software already deployed in ZON's residential cable gateways, serving more than $500.000 \mathrm{Wi}-\mathrm{Fi}$ users all over the country. It is called $Z O N @ F O N$ and allows ZON's customers to have free Internet access wherever there is a ZON@Fon hotspot and also in the rest of FON's hotspots deployed across the globe (currently more than 12MM). This relationship between the two companies dates from 2010, and continues as FON's new developments to the software are integrated onto ZON's home gateways.

The two sites were installed in real environments within ZON's and FON's commercial networks. Taking advantage of the above mentioned ZON and FON 
commercial relationship, FON provided the access points, acting as UCN gateways, to ZON, ensuring that the same equipment was used in both sites, in order to minimize the changes required to both sites. Nonetheless, both sites present different targeted users: FON gateways are located in public places while in the ZON site the gateways are in a residential area. The statistics gathered during a fixed observation period allow direct comparison between both sites.

The FON site consists on an area of one square kilometer, where the shortest distance between access points is 60 meters. As the Fonera has a signal range of 15 meters around, the pilot will not cover the mobility scenario case. Also, in some cases the Fonera is situated in a second or third floor, decreasing the signal range to the street.

ZON's pilot locations are spread around the City of Lisbon, mostly in the vicinities. They are all residential locations and the statistics will allow understanding if unidentified users tried and used the service. The selected locations are scattered according to Fig. 11, marked as stars, as those were ZON's pilot customers that volunteered to have the equipment at their houses.

The statistics concerning usage have been collected during a period of two weeks, between 9th of September 2013 and 20th of September 2013. ULOOP requires the installation of an Android plugin provided via a regular captive portal methodology. Public places show a higher volume of users thus, increasing the heterogeneity of the samples. On the other hand, residential areas have less activity but show more predictable habits and behaviors. An important aspect to consider is the group of users/devices that these deployment sites are targeting. The statistics have been collected with the help of the Google Analytics dashboard and summarize different usage reports. There are two data segments for each graph related to the FON and the ZON demo sites.

To measure the user acceptance of the ULOOP experience, ZON has conducted a small survey with the customers that agreed to enter the demo, placing the gateways at home. This survey was conducted at the end of the statistics gathering, and full details are available in a specific technical report [16]. For the questions provided and which intended to understand the satisfaction of users concerning connection, instalation, configuration, usage, a scale of 0 to 5 was used, being 0 the most negative aspect on the scale, and 5 the most positive. Moreover, the users were previously informed that this was an experience based on a proof-of-concept.

In regards to the user-friendliness of the ULOOP Android plugin, required to be part of the ULOOP UCN community, users rated such friendliness with a total of 3.33 , in a scale of 0 to 5 . Some problems were detected concerning the identification validation process, which in ULOOP is a one-step only process required to ensure liability of the involved users.

In regards to Internet access, $66 \%$ of the universe automatically obtained Internet access immediately after the configuration of the application. This is the usual process in the ULOOP UCN model, as after the initial setup, users are not required to use credentials to access the Internet, given that ULOOP identifies users via a unique mobile token, the crypto-id. Still, a total of $33 \%$ 
users that positively gained automatically required process repetition, which is a natural consequence of the fact that the ULOOP project developed a proofof-concept, and not a product.

Concerning whether or not the experience with ULOOP was better than without a UCN model, the users replied with a tendency to a positive experience in a total of 3.2 out of 5 .

The users were asked whether or not they would recommend the service to other users, and $50 \%$ replied that they would recommend the service.

ZON also asked users whether or not they would engage commercially with such a service, and $66 \%$ replied they would.

\section{Market Perspective}

In section 2.4 we have described a socio-economic analysis for UCN differentiators, towards the main stakeholders of a UCN model. The market perspective described in this section considers such analysis and goes further into an extension of the UCN model value-chain, by including the usual Internet stakeholders. Device vendors and infrastructure vendors are added to the picture as equipment and technology providers who sell both to and through operators or service providers or directly to end-users and consumers.

The exploitation and new potential business opportunities and the targeted markets are derived from the relevant features identified during the ULOOP project development [17] and, particularly, from the potential of dynamical growth of UCNs.

\subsection{Operator and Service Providers}

UCNs can be seen as such a differentiators as they enable operators to provide their customers with services that involve subscribers as consumers but also as contributors in the design and developed, i.e., community-based services. The most evident service defined in ULOOP is Internet access while roaming, but also other services can be provided such as, net

By integrating UCNs, operators may assist to the rise of new opportunities, e.g. higher customer retention and new subscriptions motivated by the UCN derived services, as well as by the possibility to offload traffic towards UCNs.

In order to succeed, the derived business models - which have as stakeholders users and operators - need to be defined in a way that revenues and other benefits are shared in a fair way across all stakeholders. For instance, an operator may propose to its specific UCN communities advertising when certain resources are exchanged (it can even be a target advertising service based on monitoring information provided by the community itself). Revenues obtained from this advertising service can be shared with the users providing the resources. Alternatively, revenues can be indirectly shared with users through discounts on subscriber invoice, increase of data tariff cap, etc. 
The opportunities here described are not simply based on hypothesis. Their effectiveness has already been partially proven in the first generation of UCNs, of which FON is an example. When FON partners with a fixed operator to deploy a community Wi-Fi (i.e. a Wi-Fi network formed by the aggregation of residential Wi-Fi access points), the operator provides the service (i.e. user roaming across the community $\mathrm{Wi}-\mathrm{Fi}$ ) at no cost for the subscriber, as a value added service. This has resulted in a significant reduction of churn (i.e. the proportion of contractual customers or subscribers who leave a supplier during a given time period), which has as consequence savings in customer retention campaigns, revenues increase coming from new and more loyal subscribers, etc.

A second opportunity related with the inclusion of UCNs as a differentiator service to the operator infrastructure can be the offer of resources to specific UCN communities. Here, a UCN community becomes a crowd-based distribution channel, not only for offering but even to validate new services, with little risk.

A third opportunity derived from the inclusion of UCNs relate with the capability to expand capillarity and capacity with minimum investment and cost. This is particularly useful to enable mobile data offloading towards UCN communities. The benefits of data offloading for operators are well known. We cite as example of an operator that profits from this opportunity $\mathrm{ZON}$, which is currently a virtual mobile operator. That means ZON is charged for any mobile traffic exchanged by its mobile subscribers.

UCNs are also relevant in the context of alternative providers. New and existing companies may arise to develop and provide UCN services regardless of the network operator(s) involved. For instance, the advertising service previously mentioned can be offered to UCN communities by an Over the Top (OTT) service provider. Alternatively, "traditional" operators may also participate in this scenario if collaboration with the OTT service provider is set up, as it happens with FON.

\subsection{Vendors}

UCNs are expected to impact the networking architectures in a way that is similar to what the Peer-to-peer (P2P) model did to services and applications. We provide the example of the ULOOP partner Huawei, that considers that some UCN concepts developed in the context of ULOOP may open opportunities for the Huawei SingleRAN solution, to provide mobile broadband services to the end user via wireless networks, from another user.

In what concerns device vendors, mobile devices are highly relevant in the context of UCNs. In this sector the main characters are PCs, laptops, video game consoles, tablets and smartphones. Nevertheless, tablets and phones are the ones that will experience the most significant impact in the context of UCNs. By the end of 2011, there were approximately 6 billion mobile subscribers on a global basis. This represents a penetration rate of approximately 87 percent based on a current global population of 7 billion. Developed countries represent 25 percent of these subscriptions with 122 percent penetration, while developing countries represent 75 percent of the subscriptions and 78 percent penetration 
[18]. The ubiquity of wireless connectivity combined with increasing functionality and speed of connected devices and mobile networks will further drive consumer demand for media, content and advertising while monetization models continue to evolve. The mobile market is complex, fragmented and both growing and evolving rapidly. Therefore, it is considered that we are in the early innings of a mobile revolution engendered by more affordable mobile computing and Internet access on a global basis.

UCNs are therefore a good opportunity in this market, as they can be seen as a social paradigm in networking, similarly to what occurred with Online Social Networks (OSNs) and content. It is expected that a similar revolution will hit the wireless network area, by allowing a community based connection that opens the doors towards new classes of applications leveraging on the user controlled network. Any device manufacturer can take an active role implementing UCN functionality in their devices. They can even create communities related to a specific device vendor, becoming an OTT service provider. This approach will allow differentiation, which is a challenge in current market scenario. Future devices with UCN capabilities will have a set of features needed in the OS of the devices and distributed with new ROMs versions.

\subsection{Wholesale Models - the FON@ZON Example}

This section provides an example on how UCN wholesale models can be devised, by relying on a specific example that involves a partnership between the ULOOP partners FON and ZON. In terms of roles in this model, FON has the role to develop, evolve, and to integrate $\mathrm{UCN}$ functionality (developed in the context of the ULOOP project) within their current technology. FON is also leading deployment, operation, and maintenance of resulting UCNs in this context. The motivation for FON is to offer derived products and services to partner operators, mobile, fixed/integrated.

ZON has the role of customer for the UCN functionality. Hence, FON is here seen as both a operator which integrates the UCN model, and a technology provider. FON acts as an OTT across the ZON owned infrastructure. While ZON is a fixed and virtual mobile operator. ZON owns broadband infrastructure where UCNs are to be integrated. As customer, ZON buys (or rents) UCN technology provided by FON and is responsible also for the deployment, operation, and maintenance of the UCNs deployed. ZON is also in charge of UCN marketing and distribution and acts as service provider towards subscribers and other potential users.

Value Proposition In this model, FON keeps on targeting telecommunications operators with UCN related products and services. FON expects to position UCNs on top of their current Community Wi-Fi offering. In this business plan example, the value proposition focus on benefits related to mobile data offloading, client retention and attraction improvement that UCN applications can enable. These benefits are crucial for an operator of the characteristics on ZON (i.e. fixed and virtual mobile operator). 
When initially introduced, the ZON@Fon service was made available to ZON customers as a free of charge, opt-in add-on to the Internet product. ZON keeps mainly targeting the residential customer as it does today, but with the perspective of an integrated solution that not only rewards users but allows them to create their own networks. Additional to the user-oriented added values of the service, it will allow the implementation of a mobile data off-loading strategy, with the corresponding savings in terms of the rent to be paid, as a MVNO. For customers, this will be an additional money-saver, from the mobile data plan they will use lesser, turning over to their UCN.

Financial Exercise Aspects This section covers revenue and cost structures and key financial indicators related to the wholesale model ZON@FON. Finally, a sensitivity analysis is presented after defining worst and best cases, in addition to the typical case. The reader must consider that these figures are based on FON and ZON experience. The aim of this exercise is to provide a structure / baseline of a business case that can be used by third parties to build their own business plans for UCN based applications.

Business Model and Revenue Structure and Expectations The business model of FON for UCN related products and services is based on a fixed and a variable fee to be charged to operators concerning licensing as well as concerning gateways [17].

Note that main inputs to derive revenue expectations are number of contracts for mobile and fixed / integrated operators (since different fees are applicable), number of UCN gateways sold (individual equipment supporting UCN functionality) and number of activated licenses (in operator CPE's) split in public and residential gateways (because different prices are applicable).

As for ZON, the financial exercise considers the expected gains from the introduction of ULOOP technology, and the impacts it will represent in terms of new users and retain of present customers - sales increase and churn reduction. This exercise is done by taking into account previous experience with ZON@Fon and the feedback from users (collected at the pilot performed with real users during ULOOP R\&D project). Users are expected to value the presence of such technology onto their home gateways. An important note is that the ARPU is not calculated just with Internet service, as it is always a part of a triple-play offering - as with the mobile, where the data plans are calculated with the voice plans. Nevertheless, the gains that are considered are those coming from the difference between revenues without and with ULOOP, as well as those directly and exclusively from ULOOP-derived services. Then, it takes into account the additional costs from deploying ULOOP onto ZON gateways, which, following on our partnership with FON, derives directly from the fees FON expects to charge for the development and maintenance work, as well as the per device license fee, based on their usual business model.

A relevant assumption is that UCN technology is software based does not requiring the usage of any additional hardware, either on customer's premises or 
at ZON's. This means that the only costs that are considered are those related to software licensing fees and platform maintenance, which are charged by FON.

Revenue improvement is driven by an increase of customer acquisition and a reduction of churn, both in fixed and mobile business but with different rates. As mentioned before, this improvement is enabled by the expectation of an increase of value perceived by current subscribers and potential new customers. Then, total revenue improvement is derived from those figures and from ZON ARPU and subscribers' base expected evolution.

Sensitivity Analysis: Worst, Typical, and Best Cases In addition to the typical case shown in previous version, a sensitivity analysis covering a worst and a best case is performed for the stakeholders involved in this wholesale model. For the FON role, two variables are considered in this analysis: number of operator contracts, and number of activated ULOOP (UCN) gateways. These variables directly impact on revenue figures. In the worst case, one year delay on contracts pace and $15 \%$ less activated ULOOP gateways are considered. While in the best case, one additional contract per year (mobile and fixed / integrated operators) and $15 \%$ more activated ULOOP gateways are considered.

The revenue results for FON are provided in Fig. 8. Looking at the worst case, it shows a relevant robustness against a pessimistic scenario where contracts are not in place (until 2016 in the case of a fixed operator and until 2017 in case of a mobile operator) and the number of activated gateways is significantly less. This robustness is enabled by the previous work performed during ULOOP project, which prevents FON to start from the scratch, and by an efficient cost structure. On the other hand, best case proves that overall results can be considerably improved by an increase of operator contracts and gateway activation. This is mainly enabled by economies of scale, since $R \& D$ and development costs are shared among different contracts.

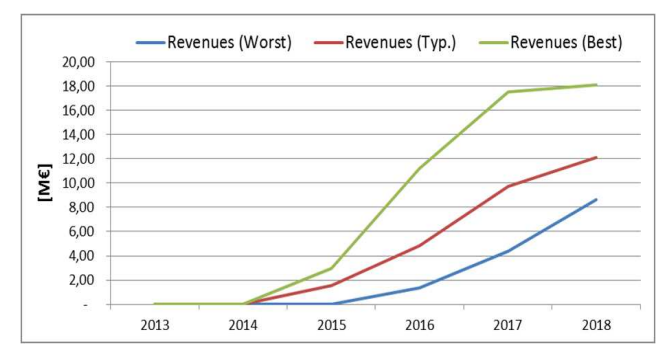

Fig. 8: FON revenue results.

As for the ZON role, variables considered are the user-sensitive components, which reflect how the customers will react to the introduction of UCNs. The pessimistic case takes the approach of a slower adoption of UCNs, reflected in a reduction in the number of new subscribers to our triple-play offering. It also 


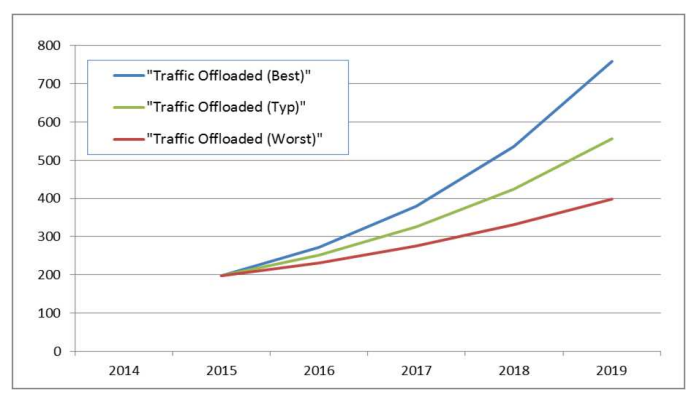

Fig. 9: ZON mobile offloading traffic impact.

considers that the deployment of UCNs will have less impact in reducing the churn. The optimistic approach considers just the opposite - that it will bring more new customers and that it will make more existing customers remain with ZON. This variation of subscribers' evolution has also a direct impact on the amount of mobile data offloaded traffic and consequently on the CAPEX savings that offloading provides, as shown in Fig. 9.

These changes on the inputs of the business case have a direct impact on revenues and costs (at a lesser extend). As explained, direct costs concerning implementation come from the FON fees for operation, maintenance and licenses. Different scenarios do not impact much in costs, due to the fact that the biggest slice of the costs comes from a fixed maintenance fee, and the per-unit fee becomes less relevant, in terms of comparing the different scenarios. Nevertheless, it is interesting to highlight that an expected decrease in the per-unit fee $\left(2016^{\sim} 2017\right)$ will, in fact and across all scenarios result in an overall reduction of the costs.

In regards to financial indicators, Fig. 10 presents the main estimates for both FON and ZON. For the case of FON, the Return of Investment (RoI) attains a better value in the worst case than in the typical one. This is because of ROI indicator inherent error. It is simply showing a better ratio between costs and net impact. Most representative and accurate indicator is the net present value and this is the one that should be taken as the main reference. Looking at the worst case, it shows a relevant robustness against a pessimistic scenario where contracts are not in place (until 2016 in the case of a fixed operator and until 2017 in case of a mobile operator) and the number of activated gateways is significantly less. This robustness is enabled by the work performed during the ULOOP project, which has as consequence the fact that FON will not start from scratch. The robustness is also enabled by an efficient cost structure. On the other hand, the best case in Fig. 10 (a) shows that overall results can be considerably improved by an increase of operator contracts and gateway activation. This is mainly enabled by economies of scale, since R\&D and development costs are shared among different contracts.

For ZON it can be understood that UCN can represent a good improvement to ZON's business, with a payback period of less than 3 years and good net 


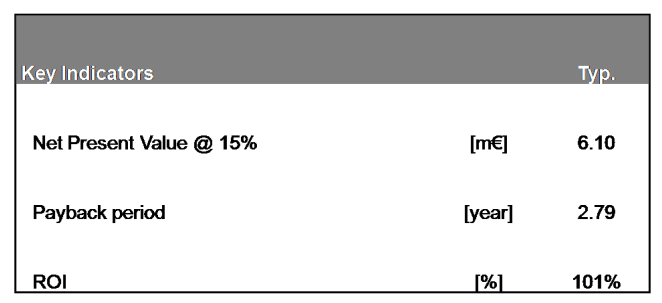

(a) FON.

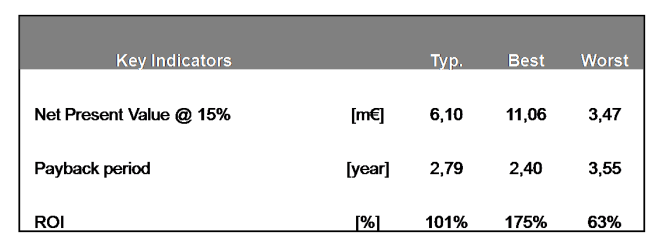

(b) ZON.

Fig. 10: Key financial indicators.

present value figure.. It is clear that the greatest improvement will not come from CAPEX savings in the mobile part, but from increases in customer base and churn reductions. As mentioned before, these are enabled because it is expected that customers perceive the value of UCNs.

\title{
6 Conclusions and Future Research
This chapter is dedicated to raise awareness, as well as to give insight into the
growing trend of user-centricity in the form of user-centric networking mod-
els. The chapter provides notions and concepts concerning UCNs, including de-
scription of existing examples, stakeholders, as well as roles in UCN models.
The chapter then debates aspects concerning cooperation models and incen-
tives for sharing in the context of wireless networks, proposing to revisit current
paradigms to consider heterogeneous exchange of resources. An operational per-
spective of UCNs is provided via a description of a specific UCN pilot, developed
in the context of the ULOOP project, which is available to the community. Such
description explains some of the results that were gathered during a live exper-
iment on two operational networks from ZON and FON. The chapter contains
also a market analysis for a concrete example of a potential cooperation, ex-
plaining how current Internet access stakeholders can profit from considering
wholesale models that integrate UCN concepts.
The expectations concerning UCNs are, from a business perspective, that
these networking architectures must not be overlooked. The chapter provides
market exercises that show the benefits that may arise for Internet stakeholders.
From a technological and scientific perspective, the concepts that have been so far
validated corroborate the potential that these networking architectures introduce \\ Conclusions and Future Research
This chapter is dedicated to raise awareness, as well as to give insight into the
growing trend of user-centricity in the form of user-centric networking mod-
els. The chapter provides notions and concepts concerning UCNs, including de-
scription of existing examples, stakeholders, as well as roles in UCN models.
The chapter then debates aspects concerning cooperation models and incen-
tives for sharing in the context of wireless networks, proposing to revisit current
paradigms to consider heterogeneous exchange of resources. An operational per-
spective of UCNs is provided via a description of a specific UCN pilot, developed
in the context of the ULOOP project, which is available to the community. Such
description explains some of the results that were gathered during a live exper-
iment on two operational networks from ZON and FON. The chapter contains
also a market analysis for a concrete example of a potential cooperation, ex-
plaining how current Internet access stakeholders can profit from considering
wholesale models that integrate UCN concepts.
The expectations concerning UCNs are, from a business perspective, that
these networking architectures must not be overlooked. The chapter provides
market exercises that show the benefits that may arise for Internet stakeholders.
From a technological and scientific perspective, the concepts that have been so far
validated corroborate the potential that these networking architectures introduce \\ Conclusions and Future Research
This chapter is dedicated to raise awareness, as well as to give insight into the
growing trend of user-centricity in the form of user-centric networking mod-
els. The chapter provides notions and concepts concerning UCNs, including de-
scription of existing examples, stakeholders, as well as roles in UCN models.
The chapter then debates aspects concerning cooperation models and incen-
tives for sharing in the context of wireless networks, proposing to revisit current
paradigms to consider heterogeneous exchange of resources. An operational per-
spective of UCNs is provided via a description of a specific UCN pilot, developed
in the context of the ULOOP project, which is available to the community. Such
description explains some of the results that were gathered during a live exper-
iment on two operational networks from ZON and FON. The chapter contains
also a market analysis for a concrete example of a potential cooperation, ex-
plaining how current Internet access stakeholders can profit from considering
wholesale models that integrate UCN concepts.
The expectations concerning UCNs are, from a business perspective, that
these networking architectures must not be overlooked. The chapter provides
market exercises that show the benefits that may arise for Internet stakeholders.
From a technological and scientific perspective, the concepts that have been so far
validated corroborate the potential that these networking architectures introduce \\ 6 Conclusions and Future Research
This chapter is dedicated to raise awareness, as well as to give insight into the
growing trend of user-centricity in the form of user-centric networking mod-
els. The chapter provides notions and concepts concerning UCNs, including de-
scription of existing examples, stakeholders, as well as roles in UCN models.
The chapter then debates aspects concerning cooperation models and incen-
tives for sharing in the context of wireless networks, proposing to revisit current
paradigms to consider heterogeneous exchange of resources. An operational per-
spective of UCNs is provided via a description of a specific UCN pilot, developed
in the context of the ULOOP project, which is available to the community. Such
description explains some of the results that were gathered during a live exper-
iment on two operational networks from ZON and FON. The chapter contains
also a market analysis for a concrete example of a potential cooperation, ex-
plaining how current Internet access stakeholders can profit from considering
wholesale models that integrate UCN concepts.
The expectations concerning UCNs are, from a business perspective, that
these networking architectures must not be overlooked. The chapter provides
market exercises that show the benefits that may arise for Internet stakeholders.
From a technological and scientific perspective, the concepts that have been so far
validated corroborate the potential that these networking architectures introduce \\ 6 Conclusions and Future Research
This chapter is dedicated to raise awareness, as well as to give insight into the
growing trend of user-centricity in the form of user-centric networking mod-
els. The chapter provides notions and concepts concerning UCNs, including de-
scription of existing examples, stakeholders, as well as roles in UCN models.
The chapter then debates aspects concerning cooperation models and incen-
tives for sharing in the context of wireless networks, proposing to revisit current
paradigms to consider heterogeneous exchange of resources. An operational per-
spective of UCNs is provided via a description of a specific UCN pilot, developed
in the context of the ULOOP project, which is available to the community. Such
description explains some of the results that were gathered during a live exper-
iment on two operational networks from ZON and FON. The chapter contains
also a market analysis for a concrete example of a potential cooperation, ex-
plaining how current Internet access stakeholders can profit from considering
wholesale models that integrate UCN concepts.
The expectations concerning UCNs are, from a business perspective, that
these networking architectures must not be overlooked. The chapter provides
market exercises that show the benefits that may arise for Internet stakeholders.
From a technological and scientific perspective, the concepts that have been so far
validated corroborate the potential that these networking architectures introduce \\ 6 Conclusions and Future Research
This chapter is dedicated to raise awareness, as well as to give insight into the
growing trend of user-centricity in the form of user-centric networking mod-
els. The chapter provides notions and concepts concerning UCNs, including de-
scription of existing examples, stakeholders, as well as roles in UCN models.
The chapter then debates aspects concerning cooperation models and incen-
tives for sharing in the context of wireless networks, proposing to revisit current
paradigms to consider heterogeneous exchange of resources. An operational per-
spective of UCNs is provided via a description of a specific UCN pilot, developed
in the context of the ULOOP project, which is available to the community. Such
description explains some of the results that were gathered during a live exper-
iment on two operational networks from ZON and FON. The chapter contains
also a market analysis for a concrete example of a potential cooperation, ex-
plaining how current Internet access stakeholders can profit from considering
wholesale models that integrate UCN concepts.
The expectations concerning UCNs are, from a business perspective, that
these networking architectures must not be overlooked. The chapter provides
market exercises that show the benefits that may arise for Internet stakeholders.
From a technological and scientific perspective, the concepts that have been so far
validated corroborate the potential that these networking architectures introduce \\ 6 Conclusions and Future Research
This chapter is dedicated to raise awareness, as well as to give insight into the
growing trend of user-centricity in the form of user-centric networking mod-
els. The chapter provides notions and concepts concerning UCNs, including de-
scription of existing examples, stakeholders, as well as roles in UCN models.
The chapter then debates aspects concerning cooperation models and incen-
tives for sharing in the context of wireless networks, proposing to revisit current
paradigms to consider heterogeneous exchange of resources. An operational per-
spective of UCNs is provided via a description of a specific UCN pilot, developed
in the context of the ULOOP project, which is available to the community. Such
description explains some of the results that were gathered during a live exper-
iment on two operational networks from ZON and FON. The chapter contains
also a market analysis for a concrete example of a potential cooperation, ex-
plaining how current Internet access stakeholders can profit from considering
wholesale models that integrate UCN concepts.
The expectations concerning UCNs are, from a business perspective, that
these networking architectures must not be overlooked. The chapter provides
market exercises that show the benefits that may arise for Internet stakeholders.
From a technological and scientific perspective, the concepts that have been so far
validated corroborate the potential that these networking architectures introduce \\ Conclusions and Future Research
This chapter is dedicated to raise awareness, as well as to give insight into the
growing trend of user-centricity in the form of user-centric networking mod-
els. The chapter provides notions and concepts concerning UCNs, including de-
scription of existing examples, stakeholders, as well as roles in UCN models.
The chapter then debates aspects concerning cooperation models and incen-
tives for sharing in the context of wireless networks, proposing to revisit current
paradigms to consider heterogeneous exchange of resources. An operational per-
spective of UCNs is provided via a description of a specific UCN pilot, developed
in the context of the ULOOP project, which is available to the community. Such
description explains some of the results that were gathered during a live exper-
iment on two operational networks from ZON and FON. The chapter contains
also a market analysis for a concrete example of a potential cooperation, ex-
plaining how current Internet access stakeholders can profit from considering
wholesale models that integrate UCN concepts.
The expectations concerning UCNs are, from a business perspective, that
these networking architectures must not be overlooked. The chapter provides
market exercises that show the benefits that may arise for Internet stakeholders.
From a technological and scientific perspective, the concepts that have been so far
validated corroborate the potential that these networking architectures introduce \\ Conclusions and Future Research
This chapter is dedicated to raise awareness, as well as to give insight into the
growing trend of user-centricity in the form of user-centric networking mod-
els. The chapter provides notions and concepts concerning UCNs, including de-
scription of existing examples, stakeholders, as well as roles in UCN models.
The chapter then debates aspects concerning cooperation models and incen-
tives for sharing in the context of wireless networks, proposing to revisit current
paradigms to consider heterogeneous exchange of resources. An operational per-
spective of UCNs is provided via a description of a specific UCN pilot, developed
in the context of the ULOOP project, which is available to the community. Such
description explains some of the results that were gathered during a live exper-
iment on two operational networks from ZON and FON. The chapter contains
also a market analysis for a concrete example of a potential cooperation, ex-
plaining how current Internet access stakeholders can profit from considering
wholesale models that integrate UCN concepts.
The expectations concerning UCNs are, from a business perspective, that
these networking architectures must not be overlooked. The chapter provides
market exercises that show the benefits that may arise for Internet stakeholders.
From a technological and scientific perspective, the concepts that have been so far
validated corroborate the potential that these networking architectures introduce \\ 6 Conclusions and Future Research
This chapter is dedicated to raise awareness, as well as to give insight into the
growing trend of user-centricity in the form of user-centric networking mod-
els. The chapter provides notions and concepts concerning UCNs, including de-
scription of existing examples, stakeholders, as well as roles in UCN models.
The chapter then debates aspects concerning cooperation models and incen-
tives for sharing in the context of wireless networks, proposing to revisit current
paradigms to consider heterogeneous exchange of resources. An operational per-
spective of UCNs is provided via a description of a specific UCN pilot, developed
in the context of the ULOOP project, which is available to the community. Such
description explains some of the results that were gathered during a live exper-
iment on two operational networks from ZON and FON. The chapter contains
also a market analysis for a concrete example of a potential cooperation, ex-
plaining how current Internet access stakeholders can profit from considering
wholesale models that integrate UCN concepts.
The expectations concerning UCNs are, from a business perspective, that
these networking architectures must not be overlooked. The chapter provides
market exercises that show the benefits that may arise for Internet stakeholders.
From a technological and scientific perspective, the concepts that have been so far
validated corroborate the potential that these networking architectures introduce \\ 6 Conclusions and Future Research
This chapter is dedicated to raise awareness, as well as to give insight into the
growing trend of user-centricity in the form of user-centric networking mod-
els. The chapter provides notions and concepts concerning UCNs, including de-
scription of existing examples, stakeholders, as well as roles in UCN models.
The chapter then debates aspects concerning cooperation models and incen-
tives for sharing in the context of wireless networks, proposing to revisit current
paradigms to consider heterogeneous exchange of resources. An operational per-
spective of UCNs is provided via a description of a specific UCN pilot, developed
in the context of the ULOOP project, which is available to the community. Such
description explains some of the results that were gathered during a live exper-
iment on two operational networks from ZON and FON. The chapter contains
also a market analysis for a concrete example of a potential cooperation, ex-
plaining how current Internet access stakeholders can profit from considering
wholesale models that integrate UCN concepts.
The expectations concerning UCNs are, from a business perspective, that
these networking architectures must not be overlooked. The chapter provides
market exercises that show the benefits that may arise for Internet stakeholders.
From a technological and scientific perspective, the concepts that have been so far
validated corroborate the potential that these networking architectures introduce \\ 6 Conclusions and Future Research
This chapter is dedicated to raise awareness, as well as to give insight into the
growing trend of user-centricity in the form of user-centric networking mod-
els. The chapter provides notions and concepts concerning UCNs, including de-
scription of existing examples, stakeholders, as well as roles in UCN models.
The chapter then debates aspects concerning cooperation models and incen-
tives for sharing in the context of wireless networks, proposing to revisit current
paradigms to consider heterogeneous exchange of resources. An operational per-
spective of UCNs is provided via a description of a specific UCN pilot, developed
in the context of the ULOOP project, which is available to the community. Such
description explains some of the results that were gathered during a live exper-
iment on two operational networks from ZON and FON. The chapter contains
also a market analysis for a concrete example of a potential cooperation, ex-
plaining how current Internet access stakeholders can profit from considering
wholesale models that integrate UCN concepts.
The expectations concerning UCNs are, from a business perspective, that
these networking architectures must not be overlooked. The chapter provides
market exercises that show the benefits that may arise for Internet stakeholders.
From a technological and scientific perspective, the concepts that have been so far
validated corroborate the potential that these networking architectures introduce \\ 6 Conclusions and Future Research
This chapter is dedicated to raise awareness, as well as to give insight into the
growing trend of user-centricity in the form of user-centric networking mod-
els. The chapter provides notions and concepts concerning UCNs, including de-
scription of existing examples, stakeholders, as well as roles in UCN models.
The chapter then debates aspects concerning cooperation models and incen-
tives for sharing in the context of wireless networks, proposing to revisit current
paradigms to consider heterogeneous exchange of resources. An operational per-
spective of UCNs is provided via a description of a specific UCN pilot, developed
in the context of the ULOOP project, which is available to the community. Such
description explains some of the results that were gathered during a live exper-
iment on two operational networks from ZON and FON. The chapter contains
also a market analysis for a concrete example of a potential cooperation, ex-
plaining how current Internet access stakeholders can profit from considering
wholesale models that integrate UCN concepts.
The expectations concerning UCNs are, from a business perspective, that
these networking architectures must not be overlooked. The chapter provides
market exercises that show the benefits that may arise for Internet stakeholders.
From a technological and scientific perspective, the concepts that have been so far
validated corroborate the potential that these networking architectures introduce \\ 6 Conclusions and Future Research
This chapter is dedicated to raise awareness, as well as to give insight into the
growing trend of user-centricity in the form of user-centric networking mod-
els. The chapter provides notions and concepts concerning UCNs, including de-
scription of existing examples, stakeholders, as well as roles in UCN models.
The chapter then debates aspects concerning cooperation models and incen-
tives for sharing in the context of wireless networks, proposing to revisit current
paradigms to consider heterogeneous exchange of resources. An operational per-
spective of UCNs is provided via a description of a specific UCN pilot, developed
in the context of the ULOOP project, which is available to the community. Such
description explains some of the results that were gathered during a live exper-
iment on two operational networks from ZON and FON. The chapter contains
also a market analysis for a concrete example of a potential cooperation, ex-
plaining how current Internet access stakeholders can profit from considering
wholesale models that integrate UCN concepts.
The expectations concerning UCNs are, from a business perspective, that
these networking architectures must not be overlooked. The chapter provides
market exercises that show the benefits that may arise for Internet stakeholders.
From a technological and scientific perspective, the concepts that have been so far
validated corroborate the potential that these networking architectures introduce \\ Conclusions and Future Research
This chapter is dedicated to raise awareness, as well as to give insight into the
growing trend of user-centricity in the form of user-centric networking mod-
els. The chapter provides notions and concepts concerning UCNs, including de-
scription of existing examples, stakeholders, as well as roles in UCN models.
The chapter then debates aspects concerning cooperation models and incen-
tives for sharing in the context of wireless networks, proposing to revisit current
paradigms to consider heterogeneous exchange of resources. An operational per-
spective of UCNs is provided via a description of a specific UCN pilot, developed
in the context of the ULOOP project, which is available to the community. Such
description explains some of the results that were gathered during a live exper-
iment on two operational networks from ZON and FON. The chapter contains
also a market analysis for a concrete example of a potential cooperation, ex-
plaining how current Internet access stakeholders can profit from considering
wholesale models that integrate UCN concepts.
The expectations concerning UCNs are, from a business perspective, that
these networking architectures must not be overlooked. The chapter provides
market exercises that show the benefits that may arise for Internet stakeholders.
From a technological and scientific perspective, the concepts that have been so far
validated corroborate the potential that these networking architectures introduce \\ 6 Conclusions and Future Research
This chapter is dedicated to raise awareness, as well as to give insight into the
growing trend of user-centricity in the form of user-centric networking mod-
els. The chapter provides notions and concepts concerning UCNs, including de-
scription of existing examples, stakeholders, as well as roles in UCN models.
The chapter then debates aspects concerning cooperation models and incen-
tives for sharing in the context of wireless networks, proposing to revisit current
paradigms to consider heterogeneous exchange of resources. An operational per-
spective of UCNs is provided via a description of a specific UCN pilot, developed
in the context of the ULOOP project, which is available to the community. Such
description explains some of the results that were gathered during a live exper-
iment on two operational networks from ZON and FON. The chapter contains
also a market analysis for a concrete example of a potential cooperation, ex-
plaining how current Internet access stakeholders can profit from considering
wholesale models that integrate UCN concepts.
The expectations concerning UCNs are, from a business perspective, that
these networking architectures must not be overlooked. The chapter provides
market exercises that show the benefits that may arise for Internet stakeholders.
From a technological and scientific perspective, the concepts that have been so far
validated corroborate the potential that these networking architectures introduce \\ 6 Conclusions and Future Research
This chapter is dedicated to raise awareness, as well as to give insight into the
growing trend of user-centricity in the form of user-centric networking mod-
els. The chapter provides notions and concepts concerning UCNs, including de-
scription of existing examples, stakeholders, as well as roles in UCN models.
The chapter then debates aspects concerning cooperation models and incen-
tives for sharing in the context of wireless networks, proposing to revisit current
paradigms to consider heterogeneous exchange of resources. An operational per-
spective of UCNs is provided via a description of a specific UCN pilot, developed
in the context of the ULOOP project, which is available to the community. Such
description explains some of the results that were gathered during a live exper-
iment on two operational networks from ZON and FON. The chapter contains
also a market analysis for a concrete example of a potential cooperation, ex-
plaining how current Internet access stakeholders can profit from considering
wholesale models that integrate UCN concepts.
The expectations concerning UCNs are, from a business perspective, that
these networking architectures must not be overlooked. The chapter provides
market exercises that show the benefits that may arise for Internet stakeholders.
From a technological and scientific perspective, the concepts that have been so far
validated corroborate the potential that these networking architectures introduce \\ 6 Conclusions and Future Research
This chapter is dedicated to raise awareness, as well as to give insight into the
growing trend of user-centricity in the form of user-centric networking mod-
els. The chapter provides notions and concepts concerning UCNs, including de-
scription of existing examples, stakeholders, as well as roles in UCN models.
The chapter then debates aspects concerning cooperation models and incen-
tives for sharing in the context of wireless networks, proposing to revisit current
paradigms to consider heterogeneous exchange of resources. An operational per-
spective of UCNs is provided via a description of a specific UCN pilot, developed
in the context of the ULOOP project, which is available to the community. Such
description explains some of the results that were gathered during a live exper-
iment on two operational networks from ZON and FON. The chapter contains
also a market analysis for a concrete example of a potential cooperation, ex-
plaining how current Internet access stakeholders can profit from considering
wholesale models that integrate UCN concepts.
The expectations concerning UCNs are, from a business perspective, that
these networking architectures must not be overlooked. The chapter provides
market exercises that show the benefits that may arise for Internet stakeholders.
From a technological and scientific perspective, the concepts that have been so far
validated corroborate the potential that these networking architectures introduce \\ Conclusions and Future Research
This chapter is dedicated to raise awareness, as well as to give insight into the
growing trend of user-centricity in the form of user-centric networking mod-
els. The chapter provides notions and concepts concerning UCNs, including de-
scription of existing examples, stakeholders, as well as roles in UCN models.
The chapter then debates aspects concerning cooperation models and incen-
tives for sharing in the context of wireless networks, proposing to revisit current
paradigms to consider heterogeneous exchange of resources. An operational per-
spective of UCNs is provided via a description of a specific UCN pilot, developed
in the context of the ULOOP project, which is available to the community. Such
description explains some of the results that were gathered during a live exper-
iment on two operational networks from ZON and FON. The chapter contains
also a market analysis for a concrete example of a potential cooperation, ex-
plaining how current Internet access stakeholders can profit from considering
wholesale models that integrate UCN concepts.
The expectations concerning UCNs are, from a business perspective, that
these networking architectures must not be overlooked. The chapter provides
market exercises that show the benefits that may arise for Internet stakeholders.
From a technological and scientific perspective, the concepts that have been so far
validated corroborate the potential that these networking architectures introduce \\ Conclusions and Future Research
This chapter is dedicated to raise awareness, as well as to give insight into the
growing trend of user-centricity in the form of user-centric networking mod-
els. The chapter provides notions and concepts concerning UCNs, including de-
scription of existing examples, stakeholders, as well as roles in UCN models.
The chapter then debates aspects concerning cooperation models and incen-
tives for sharing in the context of wireless networks, proposing to revisit current
paradigms to consider heterogeneous exchange of resources. An operational per-
spective of UCNs is provided via a description of a specific UCN pilot, developed
in the context of the ULOOP project, which is available to the community. Such
description explains some of the results that were gathered during a live exper-
iment on two operational networks from ZON and FON. The chapter contains
also a market analysis for a concrete example of a potential cooperation, ex-
plaining how current Internet access stakeholders can profit from considering
wholesale models that integrate UCN concepts.
The expectations concerning UCNs are, from a business perspective, that
these networking architectures must not be overlooked. The chapter provides
market exercises that show the benefits that may arise for Internet stakeholders.
From a technological and scientific perspective, the concepts that have been so far
validated corroborate the potential that these networking architectures introduce
}

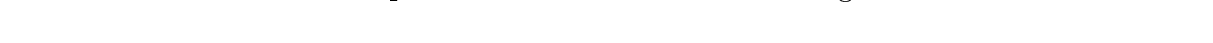

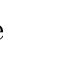


[14] A. Nosratinia, T. Hunter, and A. Hedayat, "Cooperative Communication in Wireless Networks," IEEE Communications Magazine, vol. 42, pp. 74-80, October 2004.

[15] T. Jamal, P. Mendes, and A. Zuquete, "Wireless Cooperative Relaying Based on Opportunistic Relay Selection", International Journal On Advances in Networks and Services, Vol 5, No. 1\&2, 2012.

[16] A. Matos (Editor, Caixa Mágica Software Lda), ULOOP Consortium, D4.2: Pilot Deployment and Validation. EU FP7 IST ULOOP project (grant number 257418) deliverable, October 2013.

[17] D. Valerdi (Editor, FON Wireless Ltd), ULOOP Consortium, D5.4: Exploitation Plan. EU FP7 IST ULOOP project (grant number 257418) deliverable, October 2013.

[18] Mobile Marketer: Smartphones, tablets and the mobile revolution. 29th January 2013.

[19] T. Jamal, Paulo Mendes, André Zúquete, "Analysis of Hybrid Relaying in Cooperative WLAN", in Proc. of Wireless

[20] A. Bogliolo, P. Polidori, A. Aldini, W. Moreira, P. Mendes, M. Yildiz, C. Ballester, J.-M. Seigneur . Virtual Currency and Reputation-based Cooperation Incentives. IWCMC2012.

[21] C. Ballester, J.-M.c Seigneur, P. di Francesco, V. Moreno, R. C. Sofia, Alessadro Bogliolo, N. Martins and W. M. Junior, A User-centric Approach to Trust Management in Wi-Fi Networks, IEEE INFOCOM - Demos Track, 2013.

[22] C. Ballester, J.-M. Seigneur. Dispositional Trust Self-Adaptation in User-Centric Networks. In Proc. of AINA, March 2013

[23] T. Jamal, P. Mendes, A. Zuquete, "RelaySpot: A Framework for Opportunistic Cooperative Relaying", ACCESS

[24] T. Jamal, P. Mendes, A. Zúquete "Interference-Aware Opportunistic Relay Selection", in Proc. of ACM CoNext

[25] T. Jamal, P. Mendes. Cooperative Relaying for Dynami Networks (EP13182366.8). August 2013.

[26] R. C. Sofia, P. Mendes and W. M. Junior, User-centric Networking LivingExamples and Challenges Ahead, chapter I, Springer, Lectures Notes in Social Networking, 2014.

[27] R. C. Sofia, User-centric Networking: bringing the Home Network to the Core, chapter I, User-centric Networking - Future Perspectives. to Appear, Springer, Lecture Notes on Social Networking, 2014.

[28] H. Haci, H. Zhu and J. Wang, "Resource Allocation in User-Centric Wireless Networks", VTCSpring, 2012.

[29] H. Haci. Novel Scheduling for a Mixture of Real-time and Non-real-time Traffic. IEEE Globecom 2012, September 2012.

[30] H. Osman, H. Zhu, H. Ha i, L. Lopes, R. So a. Method and Apparatus for communication in a wireless network (EP 13191667.8), August 2013.

[31] A. Aldini, A. Bogliolo. Model Checking of Trust-Based User-Centric Cooperative Networks. AFIN 2012, July 2012.

[32] P. Mendes, W. Moreira Junior, Tauseef Jamal, Huseyin Haci and huiling zhu, Cooperative networking in user-centric networks, User-centric Networking - Future Perspectives. to Appear, Springer, Lecture Notes in Social Networking, 2014.

[33] A. Aldini, A. Bogliolo (Editors). User-centric Networking -Future Perspectives. Springer Lecture Notes in Social Networks. 2014, to Appear.

[34] D. G. Murray, E. Yoneki, J. Crowcroft, S. Hand, "The case for crowd computing," in: Proceedings of the second ACM SIGCOMM workshop on Networking, systems, and applications on mobile handhelds, August 2010. 